\title{
DDX3 is exploited by Arenaviruses to suppress type I interferons and favor their replication
}

\author{
María Eugenia Loureiro ${ }^{1, \# a}$, Andre Luiz Zorzetto-Fernandes ${ }^{1}$, Sheli Radoshitzky ${ }^{2}$, Xiaoli \\ $\mathrm{Chi}^{2}$, Simone Dallari ${ }^{1}$, Nuha Marooki ${ }^{1}$, Psylvia Lèger ${ }^{1}$, Sabrina Foscaldi ${ }^{4}$, Sonia Sharma ${ }^{3}$, \\ Nora López ${ }^{4}$, Juan Carlos de la Torre ${ }^{5}$, Sina Bavari $^{2}$ and Elina Zúñiga ${ }^{1 *}$
}

${ }^{1}$ Division of Biological Sciences, University of California San Diego, La Jolla, CA, USA.

${ }^{2}$ Molecular and Translational Sciences Division, United States Army Medical Research Institute of Infectious Diseases, Frederick, MD, USA.

${ }^{3}$ La Jolla Institute for Allergy and Immunology, La Jolla, CA, USA.

${ }^{4}$ Centro de Virología Animal, Instituto de Ciencia y Tecnología Dr. César Milstein, Consejo Nacional de Investigaciones Científicas y Técnicas, Buenos Aires, Argentina.

${ }^{5}$ The Scripps Research Institute, Department of Immunology and Microbiology, La Jolla, CA, USA.

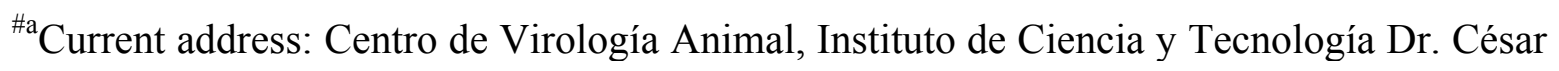
Milstein, Consejo Nacional de Investigaciones Científicas y Técnicas, Buenos Aires, Argentina.

*Correspondence to eizuniga@ucsd.edu 


\begin{abstract}
Several arenaviruses cause hemorrhagic fever (HF) diseases that are associated with high morbidity and mortality in humans. Accordingly, HF arenaviruses have been listed as top-priority emerging diseases for which countermeasures are urgently needed. Because arenavirus nucleoprotein (NP) plays critical roles in both virus multiplication and immuneevasion, we used an unbiased proteomic approach to identify NP-interacting proteins in human cells. DDX3, a DEAD-box ATP-dependent-RNA-helicase, interacted with NP in both NP-transfected and virus-infected cells. Importantly, DDX3 deficiency compromised the propagation of both Old and New World arenaviruses, including the HF arenaviruses Lassa and Junin viruses. The DDX3 role in promoting arenavirus multiplication correlated with both a previously un-recognized DDX3 contribution to type I interferon suppression in arenavirus infected cells and a positive effect of DDX3 on viral RNA synthesis. Our results uncover novel mechanisms used by arenavirus to exploit the host machinery and subvert immunity, singling out DDX3 as a potential host target for developing new therapies against highly pathogenic arenaviruses.
\end{abstract}

\title{
AUTHOR SUMMARY
}

Arenaviruses include severe clinical pathogens causing hemorrhagic fevers and have been recently incorporated by the World Health Organization in a list of critical emerging diseases for which additional research and identification of clinical targets is urgently required. A better understanding of how viral proteins interact with host cellular factors to favor arenavirus multiplication can illuminate novel pipelines on therapeutic strategies. Here we demonstrated that the ATP-dependent RNA helicase DDX3 interacted 
with the arenavirus nucleoprotein, which displays fundamental functions in different steps of the viral-cycle. Our work also revealed an unexpected new biology on the role that DDX3 might play during viral infections. In sharp contrast to previous studies showing DDX3 enhancement of IFN-I induction, we demonstrated that DDX3 suppressed IFN-I production at late time points after arenavirus infection, contributing to a DDX3 pro-viral effect. We also showed that early after infection, DDX3 pro-viral role was IFN-I independent and was mediated by DDX3 facilitation of viral RNA synthesis without affecting RNA translation. Altogether, our study established DDX3 as a critical host interacting partner of the arenavirus nucleoprotein and demonstrated two previously unrecognized DDX3-dependent strategies by which these deadly viruses exploit the host cellular machinery and suppress immunity.

\section{INTRODUCTION}

Arenaviruses include highly pathogenic hemorrhagic fever (HF) viruses endemic to West Africa and South America. Lassa virus (LASV), is an Old World (OW) arenavirus highly prevalent in West Africa where it causes about 300,000 infections and > 5,000 deaths yearly due to Lassa fever (LF), with mortality rates rising up to $50 \%$ for hospitalized patients in some outbreaks and to $90 \%$ for women in the last month of pregnancy $[1,2]$.

Notably, increased travelling has resulted in the importation of LF cases to Europe and United States, underscoring the global risk represented by this virus [3]. Likewise, several New World (NW) arenaviruses including Junin (JUNV), Machupo (MACV), Guanarito and Sabia, as well as the more recently reported Whitewater Arroyo and Chapare viruses, cause human hemorrhagic fevers with $\sim 30 \%$ mortality [4]. In addition, mounting evidence 
indicates that lymphocytic choriomeningitis virus (LCMV), a globally distributed OW arenavirus, is a neglected human pathogen that causes congenital defects and poses a special threat to immunocompromised individuals $[5,6]$. The live-attenuated vaccine Candid\#1 has been shown to be effective against Argentine HF caused by JUNV [7], but Candid\#1 is only licensed in Argentina and it does not protect against other HF arenaviral diseases. There are no other licensed arenavirus vaccines and, with the exception of the treatment with immune plasma that is restricted to JUNV infections in endemic areas [8], anti-arenaviral therapy is limited to an off-label use of the nucleoside analog ribavirin that is only partially effective [9]. Accordingly, the World Health Organization (WHO) recently included Arenaviral HF in a list of emerging diseases for which additional research and identification of clinical targets are urgently needed [10]. A better understanding of how viral proteins interact with host cellular factors to enable arenavirus propagation could aid this task.

Arenaviruses are enveloped viruses with a negative-sense RNA genome, consisting of two single-stranded segments named S (ca. $3.4 \mathrm{~kb}$ ) and L (ca. $7.2 \mathrm{~kb}$ ). The nucleoprotein (NP), encoded by the S segment, is the most abundant viral protein and plays critical roles in different steps of the arenavirus life cycle [4,11-14]. In addition, the arenavirus NP counteracts the host type I interferon (IFN-I) response during viral infection by preventing the activation and nuclear translocation of interferon regulatory factor 3 (IRF-3), and subsequent induction of IFN-I production $[15,16]$. Arenavirus-NP has also been shown to inhibit nuclear translocation and transcriptional activity of NF- $\mathrm{KB}$ [17]. The anti-IFN-I activity of arenavirus NP was mapped to its C-terminal region and was associated to a folding domain corresponding to a functional 3'-5' exonuclease of the DEDDH family $[18,19]$. NP has also been shown to interact with IKKe [20], MDA5 and RIG-I [21] which 
are involved in the IRF3 and NF-אB signaling pathways [22]. Moreover, it was recently demonstrated that NP can also subvert immune responses by associating to the dsRNAactivated protein kinase (PKR), a well-characterized antiviral protein that inhibits capdependent protein translation initiation via phosphorylation of eIF2 $\alpha$ [23]. Thus, the arenavirus NP plays essential roles in viral multiplication and the virus's ability to counteract key components of the host's antiviral innate immune response. Targeting NPhost cell protein interactions required for NP to execute its functions could facilitate novel strategies to curtail arenavirus life cycle.

In the present study, we pursued an unbiased approach to identify novel host factors targeted by the arenavirus NP that could contribute to viral multiplication or be exploited by the virus to subvert the immune response. We found that DDX3, a DEAD (Asp-GluAla-Asp)-box ATP-dependent RNA helicase, directly interacted with LCMV NP and was critical for supporting optimal LCMV growth, a finding that was extended to both OW and NW arenavirus infections in human cells. Strikingly, and in contrast to roles previously ascribed to DDX3 in promoting IFN-I [24,25], we observed that DDX3 contributed to IFNI suppression upon arenavirus infection, partially explaining its pro-viral effect late in infection. In contrast, the early pro-viral effect of DDX3 was IFN-I independent and was explained by DDX3 positive effect on viral RNA synthesis. Our results uncovered previously unrecognized maneuvers evolved in highly pathogenic arenaviruses to favor their own growth by exploiting the host machinery and evading the immune system, raising DDX3 as a potential universal target for the rational design of antiviral therapies against arenaviruses infections. 


\section{RESULTS}

\section{Unbiased identification of LASV and LCMV NP interacting proteins in human cells.}

To identify novel host proteins that could take part in protein-protein interactions with the arenavirus NP, we applied an unbiased proteomics approach. We used a human lung epithelial cell line, A549 that was transfected with plasmids expressing either LCMV or LASV NPs fused to HA tag (NP-HA). As negative controls we included cells transfected with a plasmid encoding an HA-tagged protein unrelated to the arenavirus NP but with a similar molecular weight, the ubiquitin carboxyl-terminal hydrolase 14 (HA-USP14), as well as cells infected with a newly generated recombinant tri-segmented LCMV, expressing HA-tagged GFP (3rLCMV-HA-GFP, Fig. S1A). Cell lysates were immunoprecipitated with anti-HA monoclonal antibody (Fig. S1B) followed by mass spectrometry to identify interacting peptides, using a criteria of at least 2 unique tryptic peptides with a degree of confidence of $99 \%$ to identify each hit. This approach revealed a number of NP-interacting host proteins detected with LCMV, LASV, or both, NP-HA samples but never found in negative controls (Table 1). Thus, after conducting an unbiased proteomics approach in human cells, we pinpointed a selected number of host proteins as novel candidates involved in protein-protein interactions with LCMV and/or LASV NP.

Table 1. Host proteins interacting with the NP of LCMV, LASV, or both.

\begin{tabular}{|c|l|c|c|c|c|}
\hline GI & \multicolumn{1}{|c|}{ Annotation } & LCMV & LASV & Total & NSC \\
\hline $\mathbf{4 1 3 9 9 2 8 5}$ & Chaperonin (HSPD1) & 3 & 3 & 6 & 0.03892668 \\
\hline 55956788 & Nucleolin \# & 3 & 1 & 4 & 0.011823951 \\
4506901 & Splicing factor, arginine/serine-rich 3 * & 3 & 1 & 4 & 0.004537574 \\
\hline 7705813 & Ribosomal protein L26-like 1 & 2 & 1 & 3 & 0.01543909 \\
4506633 & Ribosomal protein L31 isoform 1 & 1 & 2 & 3 & 0.012745707 \\
4506619 & Ribosomal protein L24 & 2 & 1 & 3 & 0.010694274 \\
4506623 & Ribosomal protein L27 & 2 & 1 & 3 & 0.008230397 \\
15431297 & Ribosomal protein L13 & 1 & 2 & 3 & 0.007550774 \\
4506607 & Ribosomal protein L18 & 2 & 1 & 3 & 0.005953904 \\
4506675 & Ribophorin I precursor * & 2 & 1 & 3 & 0.001225967 \\
\hline
\end{tabular}




\begin{tabular}{|c|c|c|c|c|c|}
\hline 57863257 & T-complex protein 1 isoform a & 0 & 2 & 2 & 0.020058442 \\
\hline 57013276 & Tubulin, alpha, ubiquitous \# & 1 & 1 & 2 & 0.019521338 \\
\hline 4502643 & Chaperonin containing TCP1, subunit $6 \mathrm{~A}$ isoform a & 0 & 2 & 2 & 0.01500201 \\
\hline 24234688 & Heat shock $70 \mathrm{kda}$ protein 9 precursor * & 2 & 0 & 2 & 0.014046834 \\
\hline 4506671 & Ribosomal protein P2 & 1 & 1 & 2 & 0.012759599 \\
\hline 48762932 & Chaperonin containing TCP1, subunit 8 (theta) & 0 & 2 & 2 & 0.012183282 \\
\hline 63162572 & Chaperonin containing TCP1, subunit 3 isoform a & 0 & 2 & 2 & 0.011693309 \\
\hline 4506703 & Ribosomal protein S24 isoform c & 1 & 1 & 2 & 0.011032736 \\
\hline 4506701 & Ribosomal protein $\mathbf{2 3}$ & 1 & 1 & 2 & 0.010261216 \\
\hline 4504351 & Delta globin \# & 2 & 0 & 2 & 0.010124655 \\
\hline 5453603 & Chaperonin containing TCP1, subunit 2 & 0 & 2 & 2 & 0.008933907 \\
\hline 38455427 & Chaperonin containing TCP1, subunit 4 (delta) & 0 & 2 & 2 & 0.008867607 \\
\hline 4506741 & Ribosomal protein S7 & 1 & 1 & 2 & 0.00756368 \\
\hline 4506667 & Ribosomal protein PO & 1 & 1 & 2 & 0.00526533 \\
\hline 117190254 & Heterogeneous nuclear ribonucleoprotein $\mathrm{C}$ isoform b & 1 & 1 & 2 & 0.005008034 \\
\hline 156071459 & Solute carrier family 25 , member 5 (SLC25A5) & 1 & 1 & 2 & 0.004924006 \\
\hline 4758138 & DEAD (Asp-Glu-Ala-Asp) box polypeptide 5 (DDX5) & 1 & 1 & 2 & 0.004779654 \\
\hline 5123454 & Heat shock $70 \mathrm{kda}$ protein $1 \mathrm{~A} *$ & 2 & 0 & 2 & 0.004578327 \\
\hline 4506725 & Ribosomal protein $\mathrm{S} 4$, $\mathrm{X}$-linked $\mathrm{X}$ isoform & 1 & 1 & 2 & 0.00423095 \\
\hline 45238849 & Poly (A) binding protein, cytoplasmic $3 *$ & 2 & 0 & 2 & 0.003538014 \\
\hline 4557871 & Transferrin (TF) & 2 & 0 & 2 & 0.00213227 \\
\hline 4885661 & Viral oncogene yes-1 homolog 1 (YES1) & 2 & 0 & 2 & 0.001370464 \\
\hline 16751921 & Dermcidin preproprotein (DC) & 1 & 0 & 1 & 0.119585846 \\
\hline 4759160 & Small nuclear ribonucleoprotein polypeptide D3 & 1 & 0 & 1 & 0.017718147 \\
\hline 4504347 & Alpha 1 globin (HGB) & 1 & 0 & 1 & 0.015721736 \\
\hline 5031839 & Keratin $6 A^{*}$ & 1 & 0 & 1 & 0.013994088 \\
\hline 24307939 & Chaperonin containing TCP1, subunit 5 & 0 & 1 & 1 & 0.012340921 \\
\hline 5453607 & Chaperonin containing TCP1, subunit 7 isoform a & 0 & 1 & 1 & 0.011736379 \\
\hline 119703753 & Keratin 6B * & 1 & 0 & 1 & 0.010915491 \\
\hline 15431288 & Ribosomal protein I10a & 1 & 0 & 1 & 0.010316443 \\
\hline 58331185 & Chaperonin containing TCP 1 , subunit 7 isoform $b$ & 0 & 1 & 1 & 0.009847254 \\
\hline 4506743 & Ribosomal protein $\mathrm{S} 8$ & 0 & 1 & 1 & 0.008024566 \\
\hline 4503471 & Eukaryotic translation elongation factor 1 alpha $1 \#$ & 1 & 0 & 1 & 0.006352181 \\
\hline 5902102 & Small nuclear ribonucleoprotein D1 polypeptide $16 \mathrm{kda}$ & 1 & 0 & 1 & 0.006253464 \\
\hline 4759156 & Small nuclear ribonucleoprotein polypeptide A & 1 & 0 & 1 & 0.005277746 \\
\hline 63252906 & Tropomyosin 1 alpha chain isoform 7 (TPM) & 1 & 0 & 1 & 0.005240579 \\
\hline 17105394 & Ribosomal protein $123 a$ & 1 & 0 & 1 & 0.004703057 \\
\hline 66912162 & Histone cluster 2, h2bf \# & 1 & 0 & 1 & 0.004441802 \\
\hline 4506685 & Ribosomal protein $\mathrm{S} 13$ & 0 & 1 & 1 & 0.003684569 \\
\hline 51477708 & Heterogeneous nuclear ribonucleoprotein D isoform $d$ & 1 & 0 & 1 & 0.003543757 \\
\hline 5174431 & Ribosomal protein L10 & 1 & 0 & 1 & 0.003428397 \\
\hline 47271443 & Splicing factor, arginine/serine-rich $2 \#$ & 1 & 0 & 1 & 0.00336725 \\
\hline 76496472 & Ribosomal protein $\mathrm{L} 3$ isoform $b$ & 0 & 1 & 1 & 0.003143333 \\
\hline 57863259 & T-complex protein 1 isoform $b$ & 1 & 0 & 1 & 0.001829618 \\
\hline 55770868 & Tubulin, beta polypeptide 4 , member $Q \#$ & 1 & 0 & 1 & 0.0016905 \\
\hline 70906435 & Fibrinogen, beta chain preproprotein (FG) & 1 & 0 & 1 & 0.001515605 \\
\hline 15431310 & Keratin 14 & 1 & 0 & 1 & 0.001185735 \\
\hline 87196351 & DEAD/H (Asp-Glu-Ala-Asp/His) box polypeptide 3 (DDX3) & 1 & 0 & 1 & 0.000845418 \\
\hline 110431348 & Deleted in colorectal carcinoma (DCC) & 1 & 0 & 1 & 0.000514279 \\
\hline
\end{tabular}

Hits where considered positive when 2 unique tryptic peptides where detected in either LCMV or LASV samples and never (or only 1 tryptic peptide*) in negative controls (HA-USP14 or 3rLCMVGFP-HA). Samples belong to 4 independent experiments. Hits were first ranked in groups according to the number of times detected ( 6 to 1 ) in the 8 samples (4 LCMV + 4 LASV). Within each group, hits were ranked according to the highest NSC (Normalized spectral counts) value detected for each hit. \#Hits detected in THP1 negative controls from unrelated MS studies. Bold: Hits selected for siRNA functional screening. GI: Gene identification (NCBI databank). 


\section{Functional screening with NP interacting candidates singled out DDX3}

To functionally characterize the role of newly identified NP-interacting candidates in arenavirus infection, we conducted a loss-of-function assay to monitor viral growth in cells treated with small interfering RNAs (siRNAs) directed against selected NP interacting candidates (Table 1, bold). To select these candidates, hits in Table 1 were prioritized by further filtering out proteins for which only one unique tryptic peptide was detected in negative control samples, proteins that were detected in THP1 negative control cells from previous unrelated mass spectrometry studies, as well as arbitrarily excluding ribosomal and ribonucleoproteins. A549 cells were incubated with targeting siRNA or scrambled siRNA (Scr1-siRNA or Scr2-siRNA) prior to LCMV infection. Approximately $90 \%$ of the cells incorporated siRNA oligonucleotides (Fig. S1C) and cell viability was comparable for all siRNAs tested (Fig. S1D, representative result is shown). Cells transfected with DDX3specific, but not scrambled, siRNA showed both reduced levels of DDX3 protein (Fig. $\mathrm{S} 1 \mathrm{E})$ and a reduction of LCMV titers (i.e. $\sim 0.5 \mathrm{log}$ ) (Fig. 1A). In contrast, viral yields were unaffected upon transfection with siRNAs against other candidates. Note that a negative result in this screen could have resulted from a non-essential role of the target gene and/or insufficient down-regulation of the target gene in the conditions used in our assay. Overall, these results singled-out DDX3 as a host factor that could potentially play a pro-viral role in arenavirus life cycle.

\section{DDX3 associated to LCMV NP under both transfection and infection conditions.}

We next attempted to validate arenavirus NP interaction with DDX3. For that we transfected cells with plasmids encoding LCMV or LASV NPs, and as negative controls we used cells transfected with HA-USP14 or infected with 3rLCMV-HA-GFP. Cell lysates 
were immunoprecipitated with an anti-HA mAb, and analyzed by immunoblotting with an anti-DDX3 Ab. DDX3 levels were higher in immunoprecipates from cells transfected with LCMV or LASV NP-HA than in the negative control samples (Fig. 1B, top panel). Similar levels of DDX3 and GAPDH were detected in input lysates from all samples (Fig. 1B, middle and bottom panels). These results validated that DDX3 interacted with both LCMV and LASV NPs in transfected cells. To test whether NP and DDX3 interact in the context of arenavirus infection, we generated a recombinant LCMV expressing a HA-tagged version of NP (Fig. 1C). Viral titers of rLCMV-NP-HA were typically $\sim 1$ log lower than those obtained with WT rLCMV, but no differences in size or shape of plaques were observed and importantly both viruses replicated to similar titers in vivo, revealing no gross changes in viral fitness (Fig. S1F). DDX3 was immunoprecipitated from cells infected with rLCMV-NP-HA but not from control samples infected with 3rLCMV-HA-GFP (Fig 1D top panel). Partially cleaved NP-HA and GFP-HA in immunoprecipitates and DDX3 levels in input samples were readily detectable (Fig. 1D, middle and bottom panels). Together these results demonstrated that DDX3 associated with OW arenavirus NP both in transfected and infected cells.

\section{DDX3 promoted LCMV and LASV growth in human cells.}

To further investigate the role of DDX3 in OW arenavirus infection we generated two DDX3 knockout (ko) cell lines by using different non-overlapping RNA guides and CRISPR/Cas9 gene editing, and processed in parallel a control cell line transfected with a plasmid lacking RNA-guides (WT-pCas9). Immunoblot of cell lysates showed that DDX3 was undetectable in both ko cell lines (Fig. S2A). To investigate the impact of DDX3 gene deletion on LCMV viral growth, the DDX3 ko cell lines, WT and WT-pCas9 control cells 
were infected with LCMV and viral RNA synthesis and production of infectious progeny were monitored over time. Cell viability at the time of infection was similar for all cell lines (Fig. S2B). Instead, production of LCMV infectious progeny was largely reduced (i.e. $\sim 2$ $\log$ ) at all times examined, which correlated with reduced levels of viral RNA in DDX3 ko cells at 8 and 24 hpi (Fig 1E \& S2C). Conversely, when DDX3 ko cells were infected with Sendai virus (SeV) we did not observe any reduction in viral RNA (Fig. S2D). Importantly, reconstitution of DDX3 protein expression in DDX3 ko cells transduced with a retrovirus (RV) encoding DDX3 (Fig. S2E) resulted in significantly increased viral RNA levels compared to DDX3 ko cells transduced with empty RV (Fig. 1F). Overexpression of DDX3 in WT cells transduced with DDX3-RV did not, however, cause any significant changes in viral RNA amounts respect to WT cells transduced with empty RV (Fig 1F, blue bars). These data supported that reduced LCMV growth in DDX3 ko cells was due to lack of DDX3 expression rather than off-target effects.

We next evaluated the role of DDX3 during infection with the HF OW arenavirus LASV. For that, we performed similar infection experiments in WT versus DDX3 ko cell lines in BSL-4. Quantification of infected cells via confocal microscopy revealed a significant reduction in LASV growth in both DDX3 ko cell lines compared to WT controls at all the M.O.I. tested (Fig. 2A\&B). Consistently, we detected 100 to 1000 -fold less LASV RNA in the culture supernatants of both DDX3 ko versus WT cell lines (Fig. 2C). LASV infection rates were increased in both DDX3 ko cell lines when DDX3 expression was reconstituted via RV transduction, reaching statistical significance at M.O.I 0.05 and 0.1 (Fig. 2D). 
Altogether, these observations provided strong evidence that DDX3 was a pro-viral host factor, which promoted optimal viral growth during infection with OW arenaviruses LCMV and LASV in human cells.

\section{DDX3 contributed to the IFN-I suppression observed upon arenavirus infection}

DDX3 was previously shown to interact with several components of the IFN-I pathway and to enhance IFN-I production [24-27]. To investigate a putative role for DDX3 in IFN-I induction after arenavirus infection we quantified IFNB transcript levels in WT and DDX3 ko cells after LCMV infection. Consistent with the potent capacity of arenaviruses to suppress IFN-I induction [15,16,28-30], IFNB was undetectable in WT cells infected with LCMV (Fig. 3A, blue line). In contrast, increasing amounts of IFNB transcript were detected in DDX3ko cells during the first 24 hours after LCMV infection (Fig. 3A, red line). While this effect was more profound at M.O.I 0.5, it was also significant at M.O.I 0.1 and 2.5 (Fig. S3A) and it was attenuated when DDX3 levels were reconstituted in DDX3 ko cells, at both 24 and 48 h.p.i (Fig 3B). Similar results were obtained when IFN-I bioactivity was quantified in the culture supernatant at 48 h.p.i (Fig. 3C). These findings are in sharp contrast to the previously reported role of DDX3 in promoting IFN-I induction $[24,25]$ and revealed for the first time a suppressive role of DDX3 on IFNB transcription, suggesting that the influence of DDX3 on IFN-I production is context dependent.

To test whether increased IFN-I levels could contribute to the diminished LCMV growth in the absence of DDX3, we incubated WT and DDX3 ko cells with anti-IFN-I receptor (IFNAR) $\mathrm{mAb}$ or isotype control before and throughout LCMV infection. We observed an 11-fold increase in LCMV titers at 72 (but not 24 or 48) h.p.i. in DDX3 ko 
cells incubated with anti-IFNAR versus isotype mAb, in contrast to a 3-fold increase when anti-IFNAR was blocked in WT cells (Fig. 3D). These results suggested that while the proviral effect of DDX3 was partially due to IFN-I suppression at late time points after infection, DDX3 pro-viral activity was independent of IFN-I signaling at early time points.

This was consistent with significant reduction of LCMV RNA levels following DDX3 depletion via DDX3-specific (compared to scrambled) siRNA in Vero cells, which naturally lack the IFN-I system [31] (Fig. S3B\&C).

Altogether these results pointed to a IFN-I independent, early mechanism involved in DDX3 enhancement of arenavirus propagation, and also revealed a previously unrecognized role of DDX3 as a suppressor of $I F N B$ transcription, partially explaining DDX3 pro-viral role late after LCMV infection.

\section{DDX3 promoted arenavirus replication/transcription but was dispensable for} translation.

Given that DDX3 is able to promote translation of both viral and cellular mRNAs $[32,33]$, we next investigated whether DDX3 also played a role in arenavirus mRNA translation. For that we used a recently reported arenavirus translation assay based on capped synthetic RNAs carrying the reporter firefly Luciferase (FLUC) open reading frame [34]. Quantification of the luciferase reporter activity after transfection of a Tacaribe (TCRV) mRNA analog, or a cell-like transcript as control, in cells treated with DDX3specific indicated that the translation of both the viral and cellular mRNA analogs was unchanged compared to control cells transfected with scrambled siRNA (Fig. 3E and S4). These results suggested that the reduction in viral growth observed in DDX3 deficient cells was unlikely due to reduced RNA translation. Thus, we next investigated DDX3 role in 
arenavirus replication/transcription by using a well-established LCMV minireplicon system that assesses the activity of the intracellularly reconstituted viral ribonucleoprotein (vRNP) responsible for directing viral RNA replication and gene transcription [12]. We used a LCMV S segment-based minireplicon where the Gaussia luciferase (Gluc) and GFP reporter genes substituted for NP and GPC genes, respectively, within the S genome RNA (MG/Gluc-GFP). Levels of GFP expression in cells transfected with MG/Gluc-GFP together with plasmids expressing the viral trans-acting factors NP and L polymerase, serve as surrogate of the vRNP activity. GFP signal showed a $60 \%$ drop in minireplicon activity in DDX3 ko cells compared to WT controls (Fig. 3F), which was significantly increased when DDX3 ko cells were transfected with a DDX3-encoding, but not empty, plasmid (Fig. $3 G)$. These results suggested that the pro-viral role of DDX3 on arenavirus multiplication was likely related to a novel function of DDX3 in promoting arenavirus replication and/or transcription.

\section{DDX3 interacted with New World arenavirus NPs and promoted JUNV growth in}

\section{human cells}

We next investigated whether DDX3-NP interaction was also conserved in NW arenaviruses. For that, we transfected cells with plasmids expressing the NP of JUNV, MACV or TCRV tagged with HA and prepared cell lysates that were examined by immunoprecipitation with anti-HA mAb followed by Immunoblot with anti-DDX3 mAb. DDX3 levels were significantly enriched in immunoprecipitates from cells transfected with plasmid encoding NW arenavirus HA-tagged NPs compared to cells transfected with empty plasmids (Fig 4A), indicating that DDX3 interacted with the NP of all the NW arenaviruses tested. We next investigated whether DDX3 also played a pro-viral role in NW arenavirus 
growth. For this we first infected the two DDX3 ko cell lines (Fig. S2A) with the vaccine strain of JUNV (Candid\#1) [7]. We observed that the number of cells infected with Candid\#1 was dramatically reduced to near-undetectable levels in both DDX3 ko cell lines versus WT controls, an effect that was maintained at all M.O.I tested (Fig. 4B). Importantly, DDX3 reconstitution resulted in a significant increase in infection rate, which reached statistical significance at M.O.I 1 (Fig. 4C). To confirm these results with a highly pathogenic strain of JUNV, both DDX3 ko cell lines were infected with JUNV Romero strain in BSL-4. Quantification of infected cells and JUNV RNA levels in cell culture supernatants indicated a dramatic decrease in both parameters in DDX3 ko versus WT control cells, regardless of the M.O.I used (Fig. 4 D-F). This effect was partly reverted in both DDX3 ko cell lines when DDX3 expression was reconstituted, reaching statistical significance at all M.O.I tested (Fig. 4G). These results indicated that, as with OW arenaviruses, the NP of NW arenaviruses interacted with DDX3 and that this helicase was required for optimal growth of JUNV in human cells.

\section{DISCUSSION}

Arenaviruses are endemic in their natural rodent hosts and often infect humans, with LASV causing thousands of lethal hemorrhagic fever cases each year [1-3]. Moreover, these viruses have been listed among the top priority emerging pathogens that are likely to cause a severe outbreak in the near future [10]. Currently there is no FDA-approved vaccine against arenavirus infections and there are limited therapeutic options that include Ribavirin, a compound with many side effects that requires administration in the first days of infection to show partial effectiveness [9]. Thus, there is an urgent need to develop new strategies to treat or prevent arenavirus infection in humans. A better understanding of 
arenavirus host interactions will not only inform about fundamental cellular processes exploited or subverted by these viruses, but could also help identify such intervention strategies. Given that therapeutic targeting of host (rather than viral) factors would minimize arenavirus escape mutants, we used an unbiased approach to identify arenavirus interacting candidates in human cells. Because of its abundant expression and key role in viral fitness and immune-evasion, we focused on host factors that interact with the viral protein NP. Among several newly identified NP interacting candidates, we established DDX3, an ATP-dependent RNA helicase, as an arenavirus target that is exploited to suppress host immunity and promote viral replication/transcription.

Notably, we have biochemically validated NP-DDX3 interaction in both NPtransfected and virus-infected cells, for both OW and NW arenavirus NPs. These results are consistent with a very recent report that examined the interactome of LCMV and JUNV NPs via mass spectrometry and documented (but did not biochemically validate or functionally characterize) DDX3 among numerous other NP interacting candidates [23]. While some of the NP-interacting proteins reported by King et al. were also detected in our NP interactome (e.g. DDX3, DDX5, SCLC25A5, HSPD-1 and TPM), other candidates were only detected by one of the two studies. These discrepancies could be related to the use of LCMV-infected cells [23] versus NP-transfected cells in our initial mass spectrometry approach, by the lower sensitivity of our method (i.e. lower spectral count values) and/or by the more stringent cut-off criteria that we used based on $99 \%$ confidence for peptide detection and absence of hits in two negative controls. Interestingly, neither we, nor King et al. [23] detected some previously documented NP-interacting host proteins including IKKe, MDA5 and RIG-I [20,21]. Most importantly, our functional studies demonstrated that DDX3 was critical for optimal arenavirus multiplication, as DDX3 
inhibition via either siRNA or CRISPR/Cas9 gene editing led to a significant reduction in viral titers after infection not only with LCMV, but also with the HF arenaviruses LASV and JUNV. Thus, although targeting DDX3 should be carefully weighed in the context of its many physiological roles [35-38], our results raised the attractive possibility that treatment with DDX3 inhibitors could be a viable and broadly-effective approach to curtail viral replication and alleviate arenavirus infections in humans, either alone or in combination with ribavirin. Interestingly, DDX3 appears to represent a convergent viral target, as it has been reported to interact with multiple types of viral proteins [24,39-42]. Although the outcome of these interactions has revealed both pro-viral and antiviral effects of DDX3, the fact that DDX3 is targeted by distantly related viruses, suggests an important role for DDX3 in antiviral defense.

In other infections a pro-viral role of DDX3 has been related to distinct mechanisms [43]. While during Japanese encephalitis virus and norovirus infections DDX3 helicase activity is crucial for viral replication via an unknown mechanism $[44,45]$, upon West Nile virus infection DDX3 is known to be sequestered from stress granules and processing bodies towards viral replication sites [46]. As for human immunodeficiency virus (HIV) infection DDX3 is important for both the nuclear export of unspliced vRNA and the translation of HIV transcripts [41,47]. Here, we showed that DDX3 deletion resulted in inhibition of arenavirus multiplication that correlated with a decreased in vRNP activity as determined in a cell-based LCMV minireplicon assay. Because DDX3 is involved in dsRNA unwinding via its helicase domain [48], it is possible that NP interacts with DDX3 to recruit into the virus replication complex a helicase activity required to facilitate RNA synthesis by the arenavirus polymerase. Alternatively, given that DDX3 is critical for stress granule (SG) formation independently of its helicase domain [49], it is also possible that 
arenavirus NP interaction with DDX3 has evolved to recruit SG (and other) proteins into the replication transcription complexes (RTC) organelles [50], thereby facilitating viral replication.

DDX3 has been reported to exert antiviral activity by enhancing IFN-I production $[51,52]$ and to up-regulate IFN-I via interaction with IKK $\varepsilon$ or TBK1 $[24,25,27]$, as well as to interact with RIG-I and MDA5 that bind to MAVS and facilitates IFN $\beta$ induction [52]. DDX3 can also act as a transcriptional regulator by interacting with IFN $\beta$ promoter [25]. Importantly, enhancement of IFN- $\beta$ production by DDX3 has been proposed to mediate its antiviral activity against vesicular stomatitis virus [52]. Very recently, DDX3 has also been reported to initiate MAVS signaling by sensing abortive RNA in HIV infected dendritic cells [51]. Strikingly, our experiments revealed a novel and unexpected role for DDX3 in suppressing IFNB transcription upon LCMV infection. Such IFN-I suppression appeared to partially contribute to DDX3 pro-viral activity late in infection, an effect that is expected to be amplified in vivo considering that studies in IFN-AR deficient animal models demonstrated critical roles played by IFN-I in promoting activation of almost all immune cells [53] and protection against arenavirus multiplication [54-56]. Although, IFNAR blockade has been shown to relieve immunosuppression during chronic LCMV infection in mice, it also enhanced viral titers initially [57,58]. The last-mentioned result is also consistent with evidence that treatment with $\mathrm{rIFN} \alpha / \beta$ early after LCMV infection promotes viral clearance [59], supporting a beneficial effect of enhancing IFN-I during arenavirus infections in vivo. It is possible that NP-sequestration of DDX3 may carry over some of the known DDX3-interacting proteins that participate in IFN-I induction (i.e. IKKe, TBK1, RIGI, MDA5 and/or MAVS) [22,53], counteracting the formation of macromolecular complexes required for IFN-I synthesis. Furthermore, the possible requirement of DDX3 in 
the formation of RTC organelles may diminish IFN-I induction by enabling the compartmentalization of viral RNA replication and transcription; therefore limiting recognition of dsRNA intermediates by innate sensing receptors. In addition, given that arenavirus mRNAs are not polyadenylated [60] and are therefore expected to be sensed by DDX3 [51], it is possible that arenavirus NP-DDX3 interaction has evolved to sequester DDX3 into compartments that are devoid of viral mRNAs [50], thereby preventing their recognition. Thus, although further studies are necessary to fully understand the underlying mechanisms, it is remarkable that deletion of a single host protein (i.e. DDX3) resulted in (at least partial) counteraction of the long-evolved capacity of arenavirus to suppress IFNB induction $[15,16,30]$.

Our findings using an unbiased proteomic approach followed by biochemical validation in infected cells, identified DDX3 as a novel interacting partner of OW and NW arenavirus NP. Importantly, we have also uncovered two previously unrecognized DDX3dependent strategies by which arenaviruses might counteract the host cell IFN-I response and exploit the host cellular machinery to maximize their multiplication. These findings provide the fundamental knowledge to consider DDX3 inhibitors as a potential therapeutic approach to treat infections by human pathogenic arenaviruses.

\section{MATERIAL AND METHODS}

Cells. A549 (Human lung epithelial cells, ATCC® CCL-185'TM, Manassas, VA), BHK-21 (Newborn Hamster kidney fibroblast cells, ATCC ${ }^{\circledR}$ CCL-10 ${ }^{\mathrm{TM}}$ ) and HEK-293T (Human epithelial kidney cells, ATCC® CRL-11268 ${ }^{\mathrm{TM}}$ ) were cultured in Dulbecco's Modified 
Eagle Medium (DMEM) (11965-118, Gibco, Grand Island, NY, USA) supplemented with

$2 \mathrm{mM}$ L-glutamine (25030081, Thermo Scientific), $50 \mathrm{U} / \mathrm{mL}$ penicillin-streptomycin (15140-163, Gibco), plus 10\% heat-inactivated FBS (Lonza). BHK-21 were also supplemented with 20\% Tryptose Phosphate Broth (18050039, Thermo Scientific). HEK293 T cells were supplemented with sodium pyruvate $(1 \mathrm{mM})$ and non-essential amino acids (0.1 mM). HEK-Blue ${ }^{\mathrm{TM}}$ IFN- $\alpha / \beta$ cell line (InvivoGen) was maintained in HEK-293T media supplemented with $10 \mu \mathrm{g} / \mathrm{ml}$ blasticidin (InvivoGen) and $200 \mu \mathrm{g} / \mathrm{ml}$ Zeocin (InvivoGen). Vero E6 cells (Cercopithecus aethiops kidney epithelial cells, ATCC® CCL-81 ${ }^{\mathrm{TM}}$ ) were cultured in Minimum Eagle Medium (MEM) (11095-080, Gibco), supplemented with 2 mM L-glutamine, $50 \mathrm{U} / \mathrm{mL}$ penicillin-streptomycin and $7.5 \%$ heat-inactivated FBS. A549, BHK-21, HEK-293T and Vero E6 cell lines were originally provided by J.C. de la Torre (The Scripps Research Institute, La Jolla, CA) and HEK-Blue ${ }^{\mathrm{TM}}$ IFN- $\alpha / \beta$ cell line by $\mathrm{S}$. Sharma (La Jolla Institute for Allergy and Immunology, La Jolla, CA)

Viruses. LCMV C113 stocks were produced in BHK-21 cells and viral titers were determined by M6 well plaque assay on Vero cells. LCMV C113 infections were performed in BSL-2 facilities as previously described [61]. All work with highly pathogenic arenaviruses was performed at the United States Army Medical Research Institute of Infectious Diseases (USAMRIID) at Fort Detrick, Frederick, MD, USA, within maximum containment (BSL-4). JUNV Romero or Candid\#1 strain and LASV Josiah strain viruses were propagated in Vero cells and viral infectivity was titrated by plaque assays as previously reported [62]. Sendai Virus infections were performed with Cantell strain. 
Generation of recombinant viruses. 3rLCMV-HA-GFP was generated by modifying the previously described 3rLCMV-GFP virus [63] through the insertion of a HA-FLAG tag sequence (YPYDVPDYADYKDDDDK) in the N-terminal end of the GFP ORF (located in place of NP ORF) in one of the pol-I S vectors by multi-fragment assembly [64] using Phusion High Fidelity Polymerase (Thermofisher Scientific). For the viral rescue, BHK-21 cells $\left(2 \times 10^{6}\right.$ cells per M6 well) were transfected for $5 \mathrm{~h}$ by using $2 \mu \mathrm{l}$ of Lipofectamine 2000 (Invitrogen) per microgram of plasmid DNA. The plasmid mixture was composed of $0.8 \mu \mathrm{g}$ of $\mathrm{pC}-\mathrm{NP}, 1 \mu \mathrm{g}$ of $\mathrm{pC}-\mathrm{L}, 1.4 \mu \mathrm{g}$ of pol-I L and $0.8 \mu \mathrm{g}$ of each of the two pol-I S vectors. We confirmed expression of HA-GFP protein $(\sim 27 \mathrm{kDA})$ in cells infected with 3rLCMV-HA-GFP by flow cytometry and Immunoblot with anti-HA Ab. Recombinant rLCMV-NP-HA was generated similarly, but using one single pol-I S vector expressing a modified NP ORF with the HA tag coding sequence on its C-terminal domain, as mentioned for 3rLCMV-HA-GFP. Primers for LCMV-HA-GFP: $1^{\text {st }}$ round (addition of FLAG seq): Fragment 1 (Fr1-Fw: CGGACATCT GGTCGACCTCCAGCATCG and Fr1Rv: GATTACAAGGATGACGACGATAAGTAA GACCCTCTGGGCCTCCCTGACTCTCCACCTCTTTCGAG) and Fragment 2 (Fr2-Fw: CTTATCGTCGTCATCCTTGTAATCCATCTTGTTGCTCAATGGTTTCTCAAGACAA ATGCGCAATCAAATGC and Fr2-Rv: CGATGCTGGAGGTCGACCAGATGTCCG). $2^{\text {nd }}$ round (addition of HA seq): Fragment 3 (Fr1-Fw and Fr3-Rv: TACCCTTATGATGTCCCAGATTATGCCGATTACAAGGATGACGACGATAAGGTG AGC) and Fragment4 (Fr4-Fw: GGCATAATCTGGGACATCATAAGGGTACATCT TGTTGCTCAATGGTTTCTCAAGACAAATGCGCAATC and Fr2-Rv). Primers for rLCMV-NP-HA: Fragment 5 (Fr5-Fw: CCTACAGAAGGATGGGTCAGATTGTGACA ATGTTTGAGGCTC and Fr5-Rv: TCCGGAGCCTACCCTTATGATGTCCCAGATTA 
TGCCTAAGACCCTCTGGGCCTCCCTGACTCTCCACCTCTTTCGAGGTGG, Fragment6 (Fr6-Fw: GGCATAATCTGGGACATCATAAGGGTAGGCTCCGGAGAGT GTCACAACATTTGGGCCTCTAAAAATTAGGTCATGTGGCAG and Fr6-Rv: GgtTGgaCtTCtCTGAgGtCAGCAAtgttCAG) and Fragment 7 (Fr7-Fw: CTGAACATTGCTGACCTCAGAGAAGTCCAACC and Fr7-Rv: GAGCCTCAAACA TTGTCACAATCTGACCCATCCTTCTGTAGG).

Plasmids. pol-I S, pol-I L, pC-L, pC-NP, as well as pCAGGS plasmids encoding LASV, JUNV, MACV and TCRV NPs are described elsewhere [16]. HA-USP14: plasmid encoding ubiquitin-specific protease 14 fused to HA epitope to its N-terminal end [65]. Plasmid expressing DDX3 was constructed by inserting DDX3 cDNA in pCIneo-HA vector in EcoRI/NotI sites as previously described [66]. pSpCas9(BB)-2A-GFP construct is described in [67].

Mice. C57BL/6 mice were purchased from The Jackson laboratory (Bar Harbor, ME). All mice were bred and maintained in a closed breeding facility and mouse handling conformed to the requirements of the National Institutes of Health and the Institutional Animal Care and Use Guidelines of UCSD. 6-8 weeks old mice were infected i.v with $5 \times 10^{6}$ PFU of rLCMV or rLCMV-NP-HA.

Antibodies. Anti-HA-Tag (C29F4) Rabbit mAb \#3724 (dilution 1:3500), Anti-GAPDH (14C10) Rabbit mAb \#2118 (1:5000), Anti-rabbit IgG, HRP-linked Antibody (1:5000) were obtained from Cell Signaling Technologies. Anti-DDX3 Rabbit Ab (1:5000, SAB3500206) was obtained from Sigma-Aldrich. Anti-IFNAR2 antibody (\#21385-1, PBL 
Interferon Source) and Isotype control IgG2a antibody (\#554126, BD Pharmingen Product) were both used at $5 \mu \mathrm{g} / \mathrm{ml}$. Anti-LASV (L-52-161-6) and anti-JUNV (GD01) antibodies were obtained from the US Army research Institute of Infectious Diseases (USAMRIID) archives (PMID: 20686043, PMID: 22607481).

Immunoprecipitation and Immunoblot. A549 cells $(100,000$ cells $/ \mathrm{ml})$ were plated on M12 wells. For Mass-spectrometry, four M12 plates were used for each experimental condition. Cells were transfected with $1 \mu \mathrm{g}$ of plasmid/well, encoding different arenavirus nucleoproteins fused to HA epitope in its C-terminal end (NP-HA [68] or plasmid encoding HA-USP14. Alternatively, cells were infected with 3rLCMV-HA-GFP with an m.o.i of 0.05. Media was replaced 6h later, and 24 h.p.t. cells were washed twice with PBS and lysed with $200 \mu \mathrm{l} /$ well of Immunoprecipitation lysis buffer (Pierce IP Lysis Buffer ${ }^{\circledR}$, Thermo Scientific), supplemented with Complete EDTA-Free Protease Inhibitor Cocktail tablet (04693159001, Roche Applied Science). When indicated, samples were treated with $100 \mu \mathrm{g} / \mathrm{mL}$ RNAseA for 20 minutes before co-immunoprecipitation. All lysates were cleared by centrifugation at $12.000 \mathrm{rpm}$ for $30 \mathrm{~min}$ at $4^{\circ} \mathrm{C}$. After protein quantification, lysates were incubated at a ratio of $1 \mathrm{mg}$ lysate $/ 50 \mu \mathrm{L}$ of resin (mouse monoclonal anti-HA antibody (clone HA-7) conjugated to agarose beads, A2095, Sigma-Aldrich), rotating overnight at $4^{\circ} \mathrm{C}$. Beads were then washed 4 times with IP Lysis Buffer and 2 times with PBS. For IP:IB experiments, co-immunoprecipitated proteins were recovered with one volume of $4 \mathrm{X}$ Laemmli sample buffer (Bio-Rad) containing 2-ME and for MassSpectrometry, with $200 \mu \mathrm{l}$ of $250 \mu \mathrm{g} / \mathrm{mL}$ HA-peptide (I2149, Sigma Aldrich) per $50 \mu \mathrm{l}$ of resin. Aliquots of eluates were resolved by 10\% SDS-PAGE, transferred to PVDF 
membranes (EMD Millipore) and blocked for $1 \mathrm{~h}$ at RT with 3\% non-fat dry milk in PBS containing $0.1 \%$ Tween-20, Membranes were probed with the desired primary antibody (incubated overnight at $4^{\circ} \mathrm{C}$ ), followed by incubation with HPR-conjugated antibody (1h at RT) and visualized using SuperSignal ${ }^{\mathrm{TM}}$ West Pico PLUS Chemiluminescent Substrate (34580, Thermo Scientific). Alternatively, gels were stained with SilverQuest ${ }^{\mathrm{TM}}$ Silver Staining Kit (LC6070, Thermo Scientific), following manufacturer's instructions.

Mass spectrometry. Proteins present in eluates were concentrated using Ultra-4, membrane PLGC Ultracel-PL (UFC801024, Amicon) and resuspended in TNE (50 mM Tris $\mathrm{pH}$ 8.0, $100 \mathrm{mM} \mathrm{NaCl}, 1 \mathrm{mM}$ EDTA) buffer. Samples were adjusted to $0.1 \%$ RapiGest SF reagent (Waters Corp.) and boiled for $5 \mathrm{~min}$, followed by addition of TCEP (Tris (2carboxyethyl) phosphine) to $1 \mathrm{mM}$ final concentration and incubation at $37^{\circ} \mathrm{C}$ for $30 \mathrm{~min}$. Samples were carboxymethylated with $0.5 \mathrm{mg} / \mathrm{ml}$ of iodoacetamide for $30 \mathrm{~min}$ at $37^{\circ} \mathrm{C}$, followed by neutralization with $2 \mathrm{mM}$ TCEP, and digested with trypsin (trypsin:protein ratio - 1:50) overnight at $37^{\circ} \mathrm{C}$. RapiGest was degraded and removed by treatment with 250 $\mathrm{mM} \mathrm{HCl}$ at $37^{\circ} \mathrm{C}$ for $1 \mathrm{~h}$, followed by centrifugation at $14,000 \mathrm{rpm}$ for $30 \mathrm{~min}$ at $4{ }^{\circ} \mathrm{C}$. The soluble fraction was applied to a C18 desalting column (Thermo Scientific, PI-87782). Desalted peptides were eluted from the C18 column into the mass spectrometer using a linear gradient (5-80\%) of ACN (Acetonitrile) at a flow rate of $250 \mu \mathrm{l} / \mathrm{min}$ for $1 \mathrm{~h}$. The buffers used to create the ACN gradient were: Buffer A $\left(98 \% \mathrm{H}_{2} \mathrm{O}, 2 \% \mathrm{ACN}, 0.1 \%\right.$ formic acid, and $0.005 \%$ TFA) and Buffer B (100\% ACN, 0.1\% formic acid, and $0.005 \%$ TFA). Analysis of desalted-peptides was performed by ultra high-pressure liquid chromatography (UPLC) coupled with tandem mass spectroscopy (LC-MS/MS) using nano-spray 
ionization. Nano-spray ionization was done using a TripleTof 5600 hybrid mass spectrometer (ABSCIEX) interfaced with nano-scale reversed-phase UPLC (Waters corporation nano ACQUITY) using a $20 \mathrm{~cm}-75$ micron ID glass capillary packed with 2.5$\mu \mathrm{m} \mathrm{C18}$ (130) $\mathrm{CSH}^{\mathrm{TM}}$ beads (Waters corporation). MS/MS data were acquired in a datadependent manner in which the MS1 data was acquired for $250 \mathrm{~ms}$ at m/z of 400 to 1250 $\mathrm{Da}$ and the MS/MS data was acquired from $\mathrm{m} / \mathrm{z}$ of 50 to 2,000 Da. The Independent data acquisition (IDA) parameters were as follows; MS1-TOF acquisition time of 250 milliseconds, followed by 50 MS2 events of 48 milliseconds acquisition time for each event. The threshold to trigger MS2 event was set to 150 counts when the ion had the charge state $+2,+3$ and +4 . The ion exclusion time was set to 4 seconds. Finally, the collected data were analyzed using Protein Pilot 4.5 (ABSCIEX) for peptide identifications. Identified proteins were considered specific when at least two or more unique tryptic peptides were detected with a degree of confidence of $99 \%$ [69], and were never present in HA-USP14 or 3rLCMV-HA-GFP negative controls. Spectral count normalization (NSC) was used to estimate the relative protein abundance as described in [70]. Hits identified in 4 independent experiments were ranked as depicted in Table 1.

siRNA. A549 cells were transfected (in triplicate) with 100nM siRNA (siGENOME Smartpool, Dharmacon) directed to each of the 12 selected hits listed in Table 1, using HiPerfect reagent (Qiagen) and media was replenished 6 hours post-transfection, according to manufacturer's protocol. As control, cells were transfected with scrambled siRNA Pool 1 (Scr1) or Pool 2 (Scr2) (Dharmacon). siRNA transfection efficiency was evaluated using siGLO RNAi control (Dharmacon) and flow cytometry (FITC channel). 
DDX3-knockout A549 cell lines. DDX3 ko-1 and DDX3 ko-2 cell lines were generated by CRISPR/Cas9-mediated genome engineering following the protocol and algorithm described by [67] A target sequence in the first (DDX3 ko-1) or fifth (DDX3 ko-2) of human DDX3 was chosen and appropriate oligonucleotides were cloned into the BbsI site of pSpCas9(BB)-2A-GFP plasmid. (Primers: DDX3X-Exon1-Fw: CACCGAGTGGAAAATGCGCTCGGGC， DDX3X-Exon1-Rv： AAACGCCCGAGC GCATTTTCCACTC and DDX3X-Exon5-Fw: CACCGCGGAGTGATTACGATGGCAT, DDX3X-Exon5-FRv: AAACATGCCATCGTAATCACTCCGC. The plasmid was transfected for 24 hours, and the GFP-positive population was sorted by single-cell flow cytometry on a 96 well culture plate using a BD FACS Aria II Cell-Sorter. As control, A549 were transfected with empty plasmid (WT-pCas9 cells). Cells were expanded, maintained for a minimum of ten passages before their use and tested for DDX3 expression by Immunoblot with the aforementioned anti-DDX3 Ab.

Cell viability. A549 cell viability was evaluated after knockdown with different siRNAs and after stable ko of DDX3 gene, prior to viral infection with LCMV, using Ghost-dye (Tombo Biosciences) and analysed using a BD LSRII Cytometer and FlowJo software (Treestar, Inc., Ashland, OR, USA).

Retroviral mediated DDX3 reconstitution. DDX3 gene was cloned into pMD145 vector and retrovirus was assembled in Phoenix-AMPHO (ATCC $\left(\right.$ CRL-3213 ${ }^{\mathrm{TM}}$ ) retrovirus packaging cell line after transfection with TransIT-293 transfection reagent (Mirus Bio LLC). The empty vector was also used as a negative control. After 48 hours, supernatant was collected, filtered and used for A549 transduction using $20 \mu \mathrm{g} / \mathrm{mL}$ DEAE/Dextran. 
Plates were centrifuged at $1200 x$ g for 40 minutes at room temperature and transduced cells were selected with $1.5 \mu \mathrm{g} / \mathrm{mL}$ Puromycin.

\section{Quantitative image-based analysis}

Virus-infected cells were fixed in $10 \%$ buffered formalin for $72 \mathrm{~h}$ and blocked in $3 \%$ bovine serum albumin-PBS for $1 \mathrm{~h}$. Cells were then stained with murine mAbs against JUNV or LASV glycoprotein (GD01, L-52-161-6 antibodies, respectively, 1:1,000 dilution in blocking solution), followed by Alexa Fluor 488-conjugated goat anti-mouse IgG (ThermoFisher) (1:1,000 dilution in blocking solution). All infected cells were also stained with Hoechst 33342 and HCS Cell Mask Red (ThermoFisher) for nuclei and cytoplasm detection, respectively. High-content quantitative imaging data were acquired and analyzed on an Opera confocal reader (model 3842 and 5025; quadruple excitation high sensitivity; Perkin-Elmer), at two exposures using a $\times 10$ air objective lens as described previously [71]. Analysis of the images was accomplished within the Opera environment using standard Acapella scripts. Nuclei and cytoplasm staining were used to determine total cell number and cell borders, respectively. Mock-infected cells were used to establish a threshold for virus-specific staining. Quantification of virus positive cells was subsequently performed based on mean fluorescent intensities in the virus-specific staining channel. Infection rates were then determined by dividing the number of virus positive cells by the total number of cells measured.

qPCR. For BSL-2 analyses total RNA was extracted using RNeasy kits (Qiagen), and reverse transcribed into cDNA using MMLV RT (Invitrogen). cDNA quantification was performed using SYBR Green PCR kits (Applied Biosystems) and a Real-Time PCR 
Detection System (ABI). For BSL-4 infections, viral RNA yields from the media were determined by qRT-PCR as previously described [71]. Briefly, RNA was extracted with Trizol (Thermo Fischer Scientific) and the Ambion Blood RNA Isolation Kit (Thermo Fischer Scientific). The assay was performed with RNA Ultra Sense one-step kit (Thermo Fisher Scientific) and TaqMan Probe (ABI, Thermo Fischer Scientific) following the manufacturer's instructions. The primers used were: LCMV-GP-Fw: CATTCACCTGGACTTTGTCAGACTC, LCMV-GP-Rv: GCAACTGCTGTGTTC CCGAAA, LCMV-NP-Fw: GCATTGTCTGGCTGTAGCTTA, LCMV-NP-Rv: CAATGACGTTGTACAAGCGC； JUNV-NP-Fw： CGCCAACTCCATCAGTTCATC， JUNV-NP-Rv: CCATGAGGAGTGTTCAACGAAA; probe JUNV NP Prb: 5-6FAMTCCCCAGATCTCCCACCTTGAAAACTG-TAMRA; LASV-GPC-Fw： GCAGTGCTG AAAGGTCTGTACAA，LASV-GPC-Rv：AGGAGGAAAGTGACCAAACCAA， probe LASV-GPC: $\quad$ 5-6FAM-TTTGCAACGTGTGGCCT-TAMRA; $\quad$ SeV-NP-Fw: TGCCCTGGAAGATGAGTTAG, SeV-NP-Rv: GCCTGTTGGTTTGTGGTAAG; huIFNb Fw: AAACTCATGAGCAGTCTGCA, huIFNb-Rv:AGGAGATCTTCAGTTTCGGAGG huGAPDH-Fw: TGATGACATCAAGAAGGTGGTGAAG and huGAPDH-Rv: TCCTTGGAGGCCATGTGGGCCAT. Serial 10 -fold dilutions of the assayed $\left(10^{2}\right.$ to $10^{7}$ copies) virus were used as standards. Relative expression levels were determined by using the comparative cycle threshold method.

Bioactive IFN-I. Human IFN-I bioactivity in tissue culture supernatants was measured with reference to a recombinant human IFN- $\beta$ standard (InvivoGen) using HEK-Blue ${ }^{\mathrm{TM}}$ IFN- $\alpha / \beta$ cell line (InvivoGen) and QUANTI-Blue ${ }^{\mathrm{TM}}$ detection reagent, following manufacture's instructions. 
Minireplicon and Translation assay. LCMV minireplicon system was assayed as described elsewhere [72]. Briefly, WT and DDX3 ko A549 cells were transiently cotransfected, using Lipofectamine 2000, with $0.6 \mu \mathrm{g}$ of pCAGGS L, $0.15 \mu \mathrm{g}$ of pCAGGS NP and $0.5 \mu \mathrm{g}$ of the dual-reporter (green fluorescent protein (GFP) and Gaussia luciferase (Gluc)) minigenome (MG) plasmid. These constructs were driven by the human polymerase-I promoter [73]. To normalize transfection efficiencies, $0.1 \mu \mathrm{g}$ of a mammalian expression vector encoding Cypridina noctiluca luciferase (Cluc) under the control of the constitutively active simian virus 40 (SV40) promoter (pSV40-Cluc; New England BioLabs), were included in the transfection mix. GFP expression was determined by fluorescence microscopy using a Leica fluorescence microscope. Microscope images were pseudocolored using Adobe Photoshop CS4 (v11.0) software and by luminometry (Gluc) using a Lumicount luminometer (Packard). Cells were also subjected to flow cytometry analysis at $72 \mathrm{~h}$ post-transfection, and percentages of GFP-positive $\left(\mathrm{GFP}^{+}\right)$cells and mean fluorescence intensities (MFI) of the FL1-gated cell population were determined using FlowJo software (Tree Star). Luciferase gene activities were determined using Biolux Gaussia and Cypridina Luciferase Assay kits (New England BioLabs) using a Lumicount luminometer (Packard). Reporter gene activation (Gluc) is indicated as fold induction over cells transfected with a negative pCAGGS empty plasmid control instead of the viral NP. The translation assay was performed as indicated in [34]. Capped synthetic RNAs were obtained by in vitro transcription from T7 promoter-controlled constructs. The virus-like mRNA (5'wt/3'wt_2), mimicking the TCRV NP mRNA comprises a 5-nt nonviral sequence preceding the viral 5'UTR, which is fused to the reporter firefly Luciferase (FLUC) open reading frame followed by the viral 3' UTR. The cell-like 5' $\beta$ Glo/3'poly(A) 
transcript bears the 5'UTR from human $\beta$-globin, and a 53-nt 3' poly(A) tail flanking the FLUC coding sequence. Briefly, HEK-293T cells were grown in 24 well plates, transfected with 50 pmol of siRNAs against DDX3 or scrambled siRNA pool 1 (Scr), and 42 hours later transfected again with $200 \mathrm{ng}$ per well of the indicated capped synthetic RNA. As internal control, $75 \mathrm{ng} /$ well of a Renilla Luciferase (RLUC)-expressing non-capped mRNA was added to the transfection mix. Following $6 \mathrm{~h}$ incubation, lysis of transfected cells and quantification of FLUC and RLUC activities on a Biotek FLx800 luminometer were performed using Dual-luciferase reporter assay system (Promega), according to the manufacturer's instructions. FLUC activity was normalized against the corresponding value of RLUC activity in each experimental condition. For each transcript, mean FLUC values (+/- standard deviation; SD) determined in depleted cells, are shown as a percentage of those in control cells, taken as $100 \%$.

Statistics. Statistical differences were determined by Student's t test or by one-way or twoway analysis of variance (ANOVA) followed by Bonferroni post-hoc analysis using the GraphPad Prism 5 software (La Jolla, CA). For the Translation assay, statistical analyses were performed using the SPSS 17.0 statistical software package (SPSS, Inc., Chicago, IL, USA).

Data availability: Mass spectrometry results were deposited in http://www.peptideatlas.org/PASS/PASS01114. 
Ethics statement. This study was carried out in strict accordance with the recommendations in the Guide for the Care and Use of Laboratory Animals of the National Institutes of Health under a protocol (S07315) approved by the Institutional Animal Care and Use Committee at the University of California, San Diego (Animal Welfare Assurance Number: D16-00020). All efforts were made to minimize suffering of animals employed in this study.

\section{ACKNOWLEDGEMENTS}

We are deeply grateful to Dr. Ellen Wehrens for very insightful feedback on our manuscript and to Dr. Eric Bennett (Division of Biological Sciences, UCSD) for thoughtful advice in designing Mass-Spectrometry experiments and providing HA-USP14 plasmid (PMID: 19615732). Plasmids encoding LASV, JUNV, MACV and TCRV NPs were generously provided by Dr. Luis Martinez-Sobrido (Rochester Medical Center, NY. USA). Plasmid encoding DDX3 was a gift from Dr. Ricardo Soto-Rifo (Facultad de Medicina, Universidad de Chile) and plasmid pMD145 and Phoenix-AMPHO cells, from Dr. Matthew Daugherty (Division of Biological Sciences, UCSD). pSpCas9(BB)-2A-GFP plasmid was obtained from the laboratory of Dr. Feng Zhang (McGovern Institute for Brain Research at MIT) via Addgene (I.D. 48138). Sendai Virus was kindly provided by Dr. Carolina López (Penn Institute for Immunology, University of Pennsylvania).

\section{REFERENCES}

1. Fisher-Hoch SP, Tomori O, Nasidi A, Perez-Oronoz GI, Fakile Y, et al. (1995) Review of cases of nosocomial Lassa fever in Nigeria: the high price of poor medical practice. BMJ 311: 857-859. 
2. Shaffer JG, Grant DS, Schieffelin JS, Boisen ML, Goba A, et al. (2014) Lassa fever in post-conflict sierra leone. PLoS Negl Trop Dis 8: e2748.

3. Fischer WA, 2nd, Wohl DA (2017) Moving Lassa Fever Research and Care Into the 21st Century. J Infect Dis 215: 1779-1781.

4. Kerber R, Reindl S, Romanowski V, Gomez RM, Ogbaini-Emovon E, et al. (2015) Research efforts to control highly pathogenic arenaviruses: a summary of the progress and gaps. J Clin Virol 64: 120-127.

5. Amman BR, Pavlin BI, Albarino CG, Comer JA, Erickson BR, et al. (2007) Pet rodents and fatal lymphocytic choriomeningitis in transplant patients. Emerg Infect Dis 13: 719-725.

6. Bonthius DJ, Wright R, Tseng B, Barton L, Marco E, et al. (2007) Congenital lymphocytic choriomeningitis virus infection: spectrum of disease. Ann Neurol 62: 347-355.

7. Ambrosio A, Saavedra M, Mariani M, Gamboa G, Maiza A (2011) Argentine hemorrhagic fever vaccines. Hum Vaccin 7: 694-700.

8. Enria DA, Briggiler AM, Sanchez Z (2008) Treatment of Argentine hemorrhagic fever. Antiviral Res 78: 132-139.

9. McCormick JB, King IJ, Webb PA, Scribner CL, Craven RB, et al. (1986) Lassa fever. Effective therapy with ribavirin. N Engl J Med 314: 20-26.

10. WHO WHO (2016) List of Blueprint priority diseases. http://www.who.int/blueprint/priority-diseases/en/.

11. Casabona JC, Levingston Macleod JM, Loureiro ME, Gomez GA, Lopez N (2009) The RING domain and the L79 residue of $\mathrm{Z}$ protein are involved in both the rescue of nucleocapsids and the incorporation of glycoproteins into infectious chimeric arenavirus-like particles. J Virol 83: 7029-7039.

12. Lee KJ, Novella IS, Teng MN, Oldstone MB, de La Torre JC (2000) NP and L proteins of lymphocytic choriomeningitis virus (LCMV) are sufficient for efficient transcription and replication of LCMV genomic RNA analogs. J Virol 74: 34703477.

13. Lopez N, Jacamo R, Franze-Fernandez MT (2001) Transcription and RNA replication of tacaribe virus genome and antigenome analogs require $\mathrm{N}$ and $\mathrm{L}$ proteins: $\mathrm{Z}$ protein is an inhibitor of these processes. J Virol 75: 12241-12251.

14. Shtanko O, Watanabe S, Jasenosky LD, Watanabe T, Kawaoka Y (2011) ALIX/AIP1 is required for NP incorporation into Mopeia virus Z-induced virus-like particles. J Virol 85: 3631-3641.

15. Martinez-Sobrido L, Zuniga EI, Rosario D, Garcia-Sastre A, de la Torre JC (2006) Inhibition of the type I interferon response by the nucleoprotein of the prototypic arenavirus lymphocytic choriomeningitis virus. J Virol 80: 9192-9199.

16. Martinez-Sobrido L, Giannakas P, Cubitt B, Garcia-Sastre A, de la Torre JC (2007) Differential inhibition of type I interferon induction by arenavirus nucleoproteins. $\mathrm{J}$ Virol 81: 12696-12703.

17. Rodrigo WW, Ortiz-Riano E, Pythoud C, Kunz S, de la Torre JC, et al. (2012) Arenavirus nucleoproteins prevent activation of nuclear factor kappa B. J Virol 86: 8185-8197.

18. Hastie KM, Kimberlin CR, Zandonatti MA, MacRae IJ, Saphire EO (2011) Structure of the Lassa virus nucleoprotein reveals a dsRNA-specific 3' to 5' exonuclease activity essential for immune suppression. Proc Natl Acad Sci U S A 108: 2396-2401. 
19. Qi X, Lan S, Wang W, Schelde LM, Dong H, et al. (2010) Cap binding and immune evasion revealed by Lassa nucleoprotein structure. Nature 468: 779-783.

20. Pythoud C, Rodrigo WW, Pasqual G, Rothenberger S, Martinez-Sobrido L, et al. (2012) Arenavirus nucleoprotein targets interferon regulatory factor-activating kinase IKKepsilon. J Virol 86: 7728-7738.

21. Zhou S, Cerny AM, Zacharia A, Fitzgerald KA, Kurt-Jones EA, et al. (2010) Induction and inhibition of type I interferon responses by distinct components of lymphocytic choriomeningitis virus. J Virol 84: 9452-9462.

22. Wu J, Chen ZJ (2014) Innate immune sensing and signaling of cytosolic nucleic acids. Annu Rev Immunol 32: 461-488.

23. King BR, Hershkowitz D, Eisenhauer PL, Weir ME, Ziegler CM, et al. (2017) A Map of the Arenavirus Nucleoprotein-Host Protein Interactome Reveals that Junin Virus Selectively Impairs the Antiviral Activity of Double-Stranded RNA-Activated Protein Kinase (PKR). J Virol 91.

24. Schroder M, Baran M, Bowie AG (2008) Viral targeting of DEAD box protein 3 reveals its role in TBK1/IKKepsilon-mediated IRF activation. EMBO J 27: 21472157.

25. Soulat D, Burckstummer T, Westermayer S, Goncalves A, Bauch A, et al. (2008) The DEAD-box helicase DDX3X is a critical component of the TANK-binding kinase 1-dependent innate immune response. EMBO J 27: 2135-2146.

26. Oshiumi H, Ikeda M, Matsumoto M, Watanabe A, Takeuchi O, et al. (2010) Hepatitis C virus core protein abrogates the DDX3 function that enhances IPS-1-mediated IFNbeta induction. PLoS One 5: e14258.

27. Gu L, Fullam A, Brennan R, Schroder M (2013) Human DEAD box helicase 3 couples IkappaB kinase epsilon to interferon regulatory factor 3 activation. Mol Cell Biol 33: 2004-2015.

28. Carnec X, Baize S, Reynard S, Diancourt L, Caro V, et al. (2011) Lassa virus nucleoprotein mutants generated by reverse genetics induce a robust type I interferon response in human dendritic cells and macrophages. J Virol 85: 1209312097.

29. Huang Q, Shao J, Lan S, Zhou Y, Xing J, et al. (2015) In vitro and in vivo characterizations of pichinde viral nucleoprotein exoribonuclease functions. J Virol 89: 6595-6607.

30. Muller S, Geffers R, Gunther S (2007) Analysis of gene expression in Lassa virusinfected HuH-7 cells. J Gen Virol 88: 1568-1575.

31. Emeny JM, Morgan MJ (1979) Regulation of the interferon system: evidence that Vero cells have a genetic defect in interferon production. J Gen Virol 43: 247-252.

32. Lee CS, Dias AP, Jedrychowski M, Patel AH, Hsu JL, et al. (2008) Human DDX3 functions in translation and interacts with the translation initiation factor eIF3. Nucleic Acids Res 36: 4708-4718.

33. Soto-Rifo R, Rubilar PS, Limousin T, de Breyne S, Decimo D, et al. (2012) DEAD-box protein DDX3 associates with eIF4F to promote translation of selected mRNAs. EMBO J 31: 3745-3756.

34. Foscaldi S, D'Antuono A, Noval MG, de Prat Gay G, Scolaro L, et al. (2017) Regulation of Tacaribe Mammarenavirus Translation: Positive 5' and Negative 3' Elements and Role of Key Cellular Factors. J Virol 91. 
35. Ariumi Y (2014) Multiple functions of DDX3 RNA helicase in gene regulation, tumorigenesis, and viral infection. Front Genet 5: 423.

36. Lai MC, Chang WC, Shieh SY, Tarn WY (2010) DDX3 regulates cell growth through translational control of cyclin E1. Mol Cell Biol 30: 5444-5453.

37. Chang PC, Chi CW, Chau GY, Li FY, Tsai YH, et al. (2006) DDX3, a DEAD box RNA helicase, is deregulated in hepatitis virus-associated hepatocellular carcinoma and is involved in cell growth control. Oncogene 25: 1991-2003.

38. Cruciat CM, Dolde C, de Groot RE, Ohkawara B, Reinhard C, et al. (2013) RNA helicase DDX3 is a regulatory subunit of casein kinase 1 in Wnt-beta-catenin signaling. Science 339: 1436-1441.

39. Angus AG, Dalrymple D, Boulant S, McGivern DR, Clayton RF, et al. (2010) Requirement of cellular DDX3 for hepatitis $\mathrm{C}$ virus replication is unrelated to its interaction with the viral core protein. J Gen Virol 91: 122-132.

40. Owsianka AM, Patel AH (1999) Hepatitis C virus core protein interacts with a human DEAD box protein DDX3. Virology 257: 330-340.

41. Lai MC, Wang SW, Cheng L, Tarn WY, Tsai SJ, et al. (2013) Human DDX3 interacts with the HIV-1 Tat protein to facilitate viral mRNA translation. PLoS One 8: e68665.

42. Thulasi Raman SN, Liu G, Pyo HM, Cui YC, Xu F, et al. (2016) DDX3 Interacts with Influenza A Virus NS1 and NP Proteins and Exerts Antiviral Function through Regulation of Stress Granule Formation. J Virol 90: 3661-3675.

43. Valiente-Echeverria F, Hermoso MA, Soto-Rifo R (2015) RNA helicase DDX3: at the crossroad of viral replication and antiviral immunity. Rev Med Virol 25: 286-299.

44. Li C, Ge LL, Li PP, Wang Y, Dai JJ, et al. (2014) Cellular DDX3 regulates Japanese encephalitis virus replication by interacting with viral un-translated regions. Virology 449: 70-81.

45. Vashist S, Urena L, Chaudhry Y, Goodfellow I (2012) Identification of RNA-protein interaction networks involved in the norovirus life cycle. J Virol 86: 11977-11990.

46. Chahar HS, Chen S, Manjunath N (2013) P-body components LSM1, GW182, DDX3, DDX6 and XRN1 are recruited to WNV replication sites and positively regulate viral replication. Virology 436: 1-7.

47. Yedavalli VS, Neuveut C, Chi YH, Kleiman L, Jeang KT (2004) Requirement of DDX3 DEAD box RNA helicase for HIV-1 Rev-RRE export function. Cell 119: 381-392.

48. Garbelli A, Beermann S, Di Cicco G, Dietrich U, Maga G (2011) A motif unique to the human DEAD-box protein DDX3 is important for nucleic acid binding, ATP hydrolysis, RNA/DNA unwinding and HIV-1 replication. PLoS One 6: e19810.

49. Shih JW, Wang WT, Tsai TY, Kuo CY, Li HK, et al. (2012) Critical roles of RNA helicase DDX3 and its interactions with eIF4E/PABP1 in stress granule assembly and stress response. Biochem J 441: 119-129.

50. Baird NL, York J, Nunberg JH (2012) Arenavirus infection induces discrete cytosolic structures for RNA replication. J Virol 86: 11301-11310.

51. Gringhuis SI, Hertoghs N, Kaptein TM, Zijlstra-Willems EM, Sarrami-Fooroshani R, et al. (2017) HIV-1 blocks the signaling adaptor MAVS to evade antiviral host defense after sensing of abortive HIV-1 RNA by the host helicase DDX3. Nat Immunol 18: 225-235. 
52. Oshiumi H, Sakai K, Matsumoto M, Seya T (2010) DEAD/H BOX 3 (DDX3) helicase binds the RIG-I adaptor IPS-1 to up-regulate IFN-beta-inducing potential. Eur J Immunol 40: 940-948.

53. Garcia-Sastre A, Biron CA (2006) Type 1 interferons and the virus-host relationship: a lesson in detente. Science 312: 879-882.

54. Yun NE, Poussard AL, Seregin AV, Walker AG, Smith JK, et al. (2012) Functional interferon system is required for clearance of lassa virus. J Virol 86: 3389-3392.

55. Kolokoltsova OA, Yun NE, Poussard AL, Smith JK, Smith JN, et al. (2010) Mice lacking alpha/beta and gamma interferon receptors are susceptible to junin virus infection. J Virol 84: 13063-13067.

56. Ou R, Zhou S, Huang L, Moskophidis D (2001) Critical role for alpha/beta and gamma interferons in persistence of lymphocytic choriomeningitis virus by clonal exhaustion of cytotoxic T cells. J Virol 75: 8407-8423.

57. Wilson EB, Yamada DH, Elsaesser H, Herskovitz J, Deng J, et al. (2013) Blockade of chronic type I interferon signaling to control persistent LCMV infection. Science 340: 202-207.

58. Teijaro JR, Ng C, Lee AM, Sullivan BM, Sheehan KC, et al. (2013) Persistent LCMV infection is controlled by blockade of type I interferon signaling. Science 340: 207211.

59. Wang Y, Swiecki M, Cella M, Alber G, Schreiber RD, et al. (2012) Timing and magnitude of type I interferon responses by distinct sensors impact CD8 T cell exhaustion and chronic viral infection. Cell Host Microbe 11: 631-642.

60. Iapalucci S, Lopez N, Franze-Fernandez MT (1991) The 3' end termini of the Tacaribe arenavirus subgenomic RNAs. Virology 182: 269-278.

61. Ahmed R, Salmi A, Butler LD, Chiller JM, Oldstone MB (1984) Selection of genetic variants of lymphocytic choriomeningitis virus in spleens of persistently infected mice. Role in suppression of cytotoxic $\mathrm{T}$ lymphocyte response and viral persistence. J Exp Med 160: 521-540.

62. Mudhasani R, Tran JP, Retterer C, Radoshitzky SR, Kota KP, et al. (2013) IFITM-2 and IFITM-3 but not IFITM-1 restrict Rift Valley fever virus. J Virol 87: 84518464.

63. Emonet SF, Garidou L, McGavern DB, de la Torre JC (2009) Generation of recombinant lymphocytic choriomeningitis viruses with trisegmented genomes stably expressing two additional genes of interest. Proc Natl Acad Sci U S A 106: 3473-3478.

64. Gibson DG, Young L, Chuang RY, Venter JC, Hutchison CA, 3rd, et al. (2009) Enzymatic assembly of DNA molecules up to several hundred kilobases. Nat Methods 6: 343-345.

65. Sowa ME, Bennett EJ, Gygi SP, Harper JW (2009) Defining the human deubiquitinating enzyme interaction landscape. Cell 138: 389-403.

66. Pillai RS, Artus CG, Filipowicz W (2004) Tethering of human Ago proteins to mRNA mimics the miRNA-mediated repression of protein synthesis. RNA 10: 1518-1525.

67. Ran FA, Hsu PD, Wright J, Agarwala V, Scott DA, et al. (2013) Genome engineering using the CRISPR-Cas9 system. Nat Protoc 8: 2281-2308.

68. Ortiz-Riano E, Cheng BY, de la Torre JC, Martinez-Sobrido L (2012) Self-association of lymphocytic choriomeningitis virus nucleoprotein is mediated by its $\mathrm{N}$-terminal region and is not required for its anti-interferon function. J Virol 86: 3307-3317. 
69. McCormack AL, Schieltz DM, Goode B, Yang S, Barnes G, et al. (1997) Direct analysis and identification of proteins in mixtures by LC/MS/MS and database searching at the low-femtomole level. Anal Chem 69: 767-776.

70. Paoletti AC, Parmely TJ, Tomomori-Sato C, Sato S, Zhu D, et al. (2006) Quantitative proteomic analysis of distinct mammalian Mediator complexes using normalized spectral abundance factors. Proc Natl Acad Sci U S A 103: 18928-18933.

71. Radoshitzky SR, Dong L, Chi X, Clester JC, Retterer C, et al. (2010) Infectious Lassa virus, but not filoviruses, is restricted by BST-2/tetherin. J Virol 84: 10569-10580.

72. Cheng BY, Ortiz-Riano E, de la Torre JC, Martinez-Sobrido L (2015) Arenavirus Genome Rearrangement for the Development of Live Attenuated Vaccines. J Virol 89: 7373-7384.

73. Ortiz-Riano E, Cheng BY, Carlos de la Torre J, Martinez-Sobrido L (2013) Arenavirus reverse genetics for vaccine development. J Gen Virol 94: 1175-1188.

\section{LEGENDS TO FIGURES}

Figure 1. DDX3 interacted with LASV and LCMV NPs and promoted LCMV growth

in human cells. A. A549 cells were transfected for $60 \mathrm{~h}$ with targeting siRNAs specific for DDX3 or scrambled siRNA controls followed by infection with LCMV Cl13 (M.O.I. 0.005). Viral titers in cell culture supernatants harvested 24 and 72 h.p.i. are shown. B. A549 cells were transfected for $24 \mathrm{~h}$ with plasmids encoding LCMV-NP-HA, LASV-NPHA or HA-USP14, or infected with 3rLCMV-HA-GFP, lysed and immunoprecipitated with anti-HA agarose beads (IP HA); eluates were analyzed by Immunoblot (IB). Immunoblots with anti-DDX3 and anti-GAPDH (load control) Abs were performed in input samples. C. Schematics of the genome of rLCMV-NP-HA. White: ORFs of viral proteins. Pink: HA tag. Black : viral untranslated regions. D. A549 cells were infected with rLCMV-NP-HA or 3rLCMV-HA-GFP for 24h, lysed in buffer containing RNAseA $0.1 \mathrm{mg} / \mathrm{ml}$, immunoprecipitated (IP) and analyzed by Immunoblot (IB). > indicates NP-HA band. E. Viral titers in supernatants from DDX3 ko-1, DDX3 ko-2, WT-pCas9 (control) and WT A549 cells infected with LCMV C113 (M.O.I. 0.5) were quantified at 24, 48 and 72 h.p.i. F. 
DDX3 ko-1 and WT A549 cells transduced with RV expressing DDX3 or empty-RV were infected with LCMV C113 (M.O.I. 0.5) for 24h and viral RNA levels (lcmvgp) were determined relative to gapdh by RT-qPCR. Data are representative of $2(\mathbf{A}, \mathbf{D}$ and $\mathbf{F})$ or 4 (B and E) independent experiments. * $\mathrm{p}<0.05, * * \mathrm{p}<0.01, * * * \mathrm{p}<0.001$. Stars colors represent: DDX3 vs. Scr1(blue) or Scr2 (black) (A) and WT A549 vs DDX3 ko-1 (red) or vs DDX3 ko-2 (black) (E).

Figure 2. DDX3 promoted LASV growth in human cells. A to C. DDX3 ko-1, DDX3 ko-2 and WT A549 cells were infected with LASV (strain Josiah) at the indicated M.O.I. for $48 \mathrm{~h}$. A. Cells were fixed with $10 \%$ formalin for $72 \mathrm{~h}$ and stained with anti-GP antibodies and Hoechst, for confocal microscopy. Representative images are shown. B. Number of infected cells were calculated by high-content quantitative image-based analysis. C. Viral RNA yields in tissue culture supernatants were determined by qRT-PCR. D. DDX3 ko-1, DDX3 ko-2 and WT A549 cells were transduced with empty-RV (EV-RV) or RV encoding DDX3 (DDX3-RV) prior to LASV infection and then processed as in $\mathbf{B}$. All data are representative of 2 independent experiments. ${ }^{*} \mathrm{p}<0.05, * * \mathrm{p}<0.01, * * * \mathrm{p}<0.001$. Star colors represent: WT-A549 vs. DDX3 ko-1 (red) or vs. DDX3 ko-2 (black) (B) and DDX3 ko1+EV-RV vs DDX3 ko-1+DDX3-RV (red) or DDX3 ko-2+EV-RV vs DDX3 ko-2+DDX3RV (black).

Figure 3. DDX3 contributed to IFN-I suppression and viral replication/transcription after arenavirus infection in human cells. A. DDX3 ko-1, DDX3 ko-2 and WT A549 cells were infected with LCMV C113 (M.O.I. 0.5) for the indicated times. Total RNA in cell lysates was extracted and normalized viral RNA levels (ifnb/gapdh) determined by 
qRT-PCR. B-C. DDX3 ko-1 and WT A549 cells were transduced with empty-RV (EV-RV) or RV encoding DDX3 (DDX3-RV) before infection, and processed as in $\mathbf{A}$ for quantification of ifnb/gapdh transcripts via qRT-PCR (B) or determination of bioactive IFN-I levels in cell culture supernatants at indicated hp.i. (C). D. DDX3 ko-1 and WT A549 cells were pre-incubated for $2 \mathrm{~h}$ and infected with LCMV CL13 (M.O.I. 0.5) in the presence of anti-IFNAR mAb (IFNAR Ab) or Isotype control (Iso Ab), which were left for the remaining of the culture. Viral titers were determined at indicated h.p.i. E. Translation assay performed in HEK-293T cells treated with DDX3 or Scr siRNA. F. Minireplicon assay performed in DDX3 ko-1 or WT A549 cells G. DDX3 ko-1 or WT cells were transfected with $0.4 \mu \mathrm{g}$ of empty plasmid or plasmid expressing DDX3 and used for minireplicon assay. 100\% value was given to WT A549 transfected with empty plasmid. Data are representative of $2(\mathbf{A}-\mathbf{D} \& \mathbf{F}-\mathbf{G})$ or $3(\mathbf{E})$ independent experiments. $* \mathrm{p}<0.05, * *$ $\mathrm{p}<0.01, * * * \mathrm{p}<0.001$. Stars represent: DDX3ko vs WT (blue) or vs DDX3 ko at the indicated h.p.i. versus time=0 (red) (A), DDX3 ko-EV vs WT-EV (black) or vs DDX3 koRVDDX3 (red) (B), WT-EV vs DDX3 ko-EV (C) and DDX3 ko-IFNAR vs WT-IFNAR (black) or vs DDX3 ko-Isotype (red) (D).

Figure 4. DDX3 interacted with New World arenavirus NP and promoted JUNV growth in human cells. A. A549 cells were transfected with plasmid encoding JUNV, MACV or TCRV NP-HA for 24h, lysed and immunoprecipitated with anti-HA agarose beads (IP HA). Eluates (middle panel) or input samples (load control, lower panel) were analyzed by Immunoblotting (IB) with anti-DDX3 or anti-GAPDH, respectively. B-G. DDX3 ko-1, DDX3 ko-2 and WT A549 cells were infected with JUNV Candid 1 (B-D) or Romero (E-G) strains at the indicated M.O.I. for $24 \mathrm{~h}$. Cells were fixed with $10 \%$ formalin 
for $72 \mathrm{~h}$ and stained with anti-GP antibodies and Hoechst, for confocal microscopy. Representative images for infected cells are shown (D). Number of infected cells was determined by high-content quantitative image-based analysis (B, C, E and $\mathbf{G})$. Viral RNA yields in tissue culture supernatants were determined by qRT-PCR (F). When indicated, (C and G) DDX3 ko-1 and WT A549 cells were transduced with empty-RV (EV-RV) or RV encoding DDX3 (DDX3-RV) before infection, and processed as in $\mathbf{B}$ and $\mathbf{E}$, respectively. Data are representative of $2(\mathrm{~A}-\mathrm{F})$ or $1(\mathrm{G})$ independent experiments. ** $\mathrm{p}<0.01$, ***p $<0.001$. Star colors represent: DDX3 ko-EV vs WT-EV (black) or vs DDX3 koRVDDX3 (red) (C), WT vs DDX3ko-1(red) or vs DDX3ko-2 (black) (E) DDX3 ko-1+EVRV vs DDX3 ko-1+DDX3-RV (red) or DDX3 ko-2+EV-RV vs DDX3 ko-2+DDX3-RV (black) (G). 
bioRxiv preprint doi: https://doi.org/10.1101/224725; this version posted November 25, 2017. The copyright holder for this preprint (which was not certified by peer review) is the author/funder. All rights reserved. No reuse allowed without permission.

A

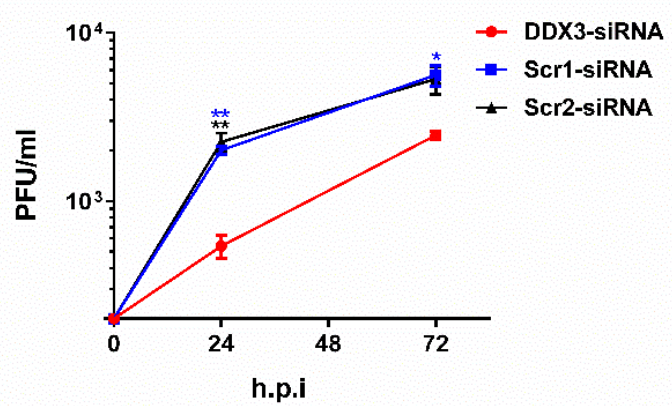

C

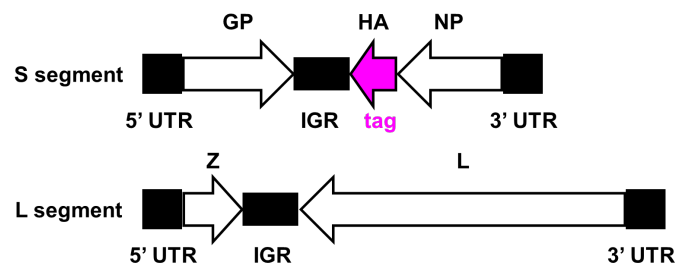

B

IP: HA

IB: DDX3

IP: HA

IB: HA

IP: -

IB: DDX3

IP: -

IB: GAPDH
LCMV LASV HA- 3rLCMV NP-HA NP-HA USP14 HA-GFP
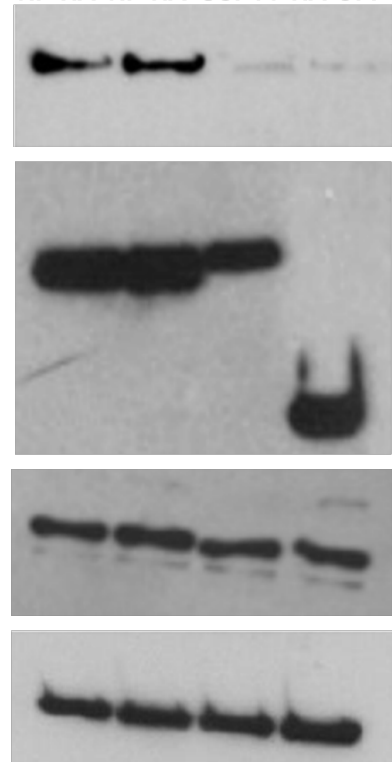

D

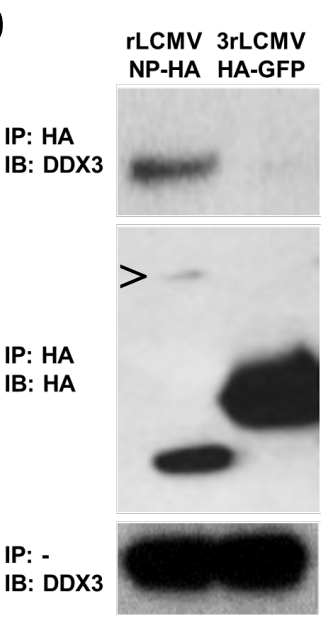

E

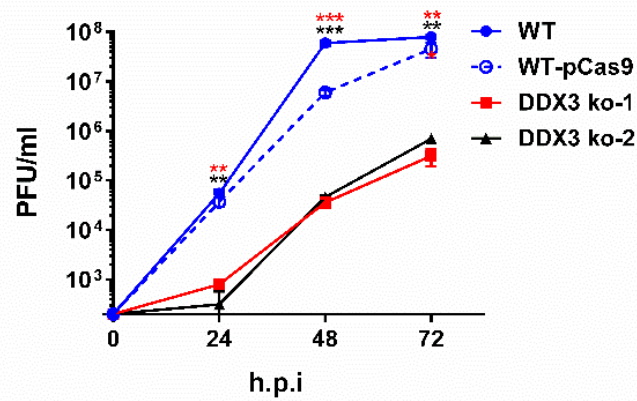

$\mathbf{F}$

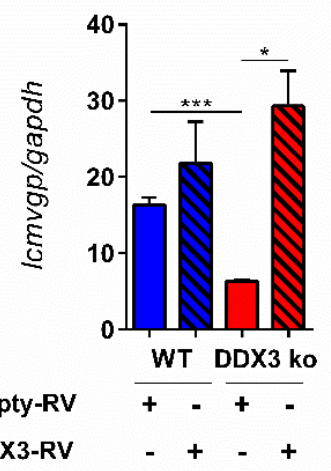

Figure 1 Loureiro et al. 

not certified by peer review) is the author/funder. All rights reserved. No reuse allowed without permission.

A

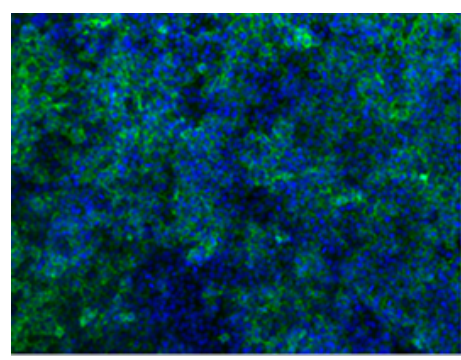

WT

B

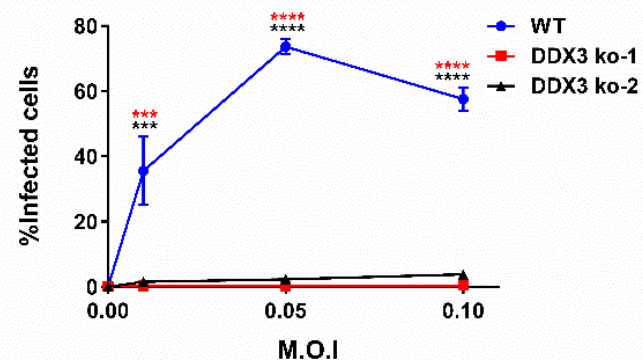

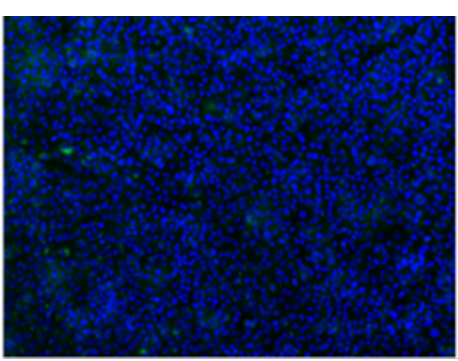

DDX3 ko-1

C

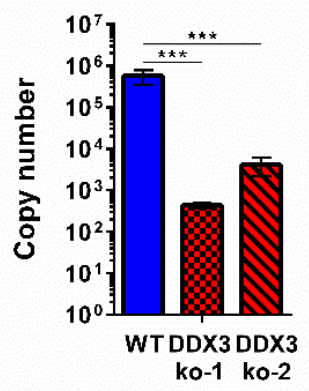

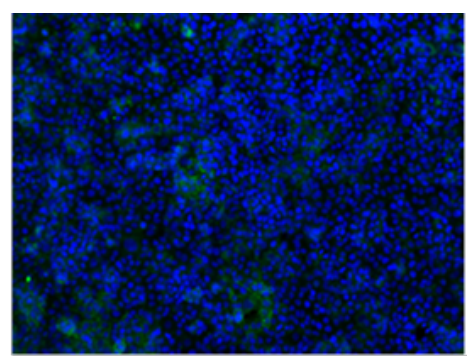

DDX3 ko-2

D

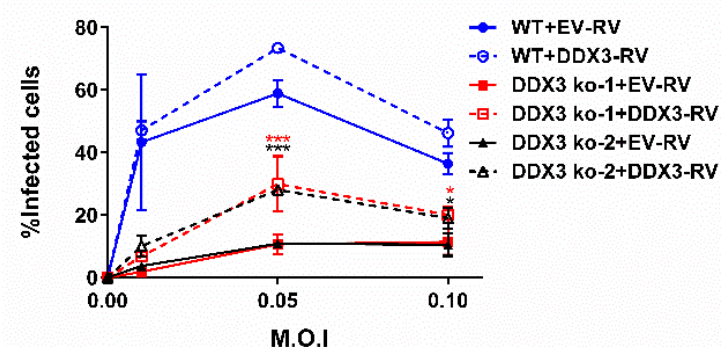


bioRxiv preprint doi: https://doi.org/10.1101/224725; this version posted November 25, 2017. The copyright holder for this preprint (which was not certified by peer review) is the author/funder. All rights reserved. No reuse allowed without permission.

A

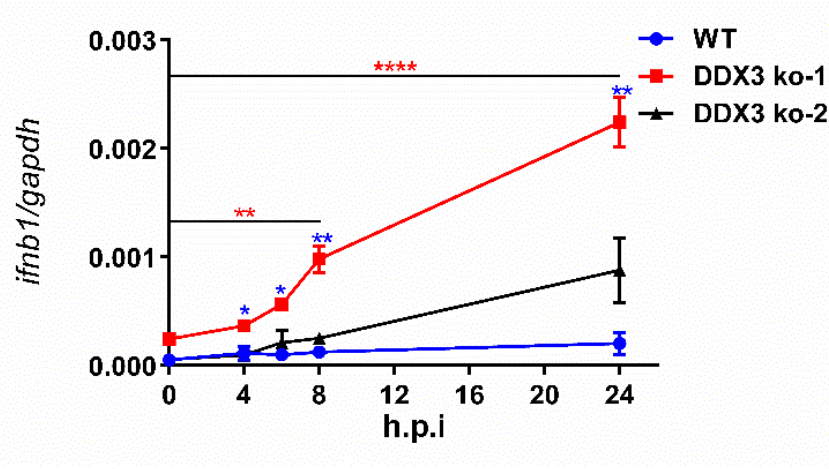

C

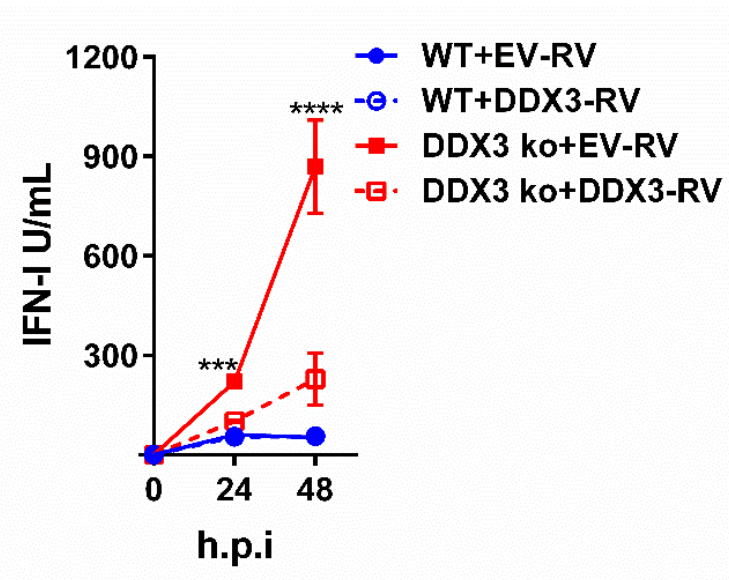

E

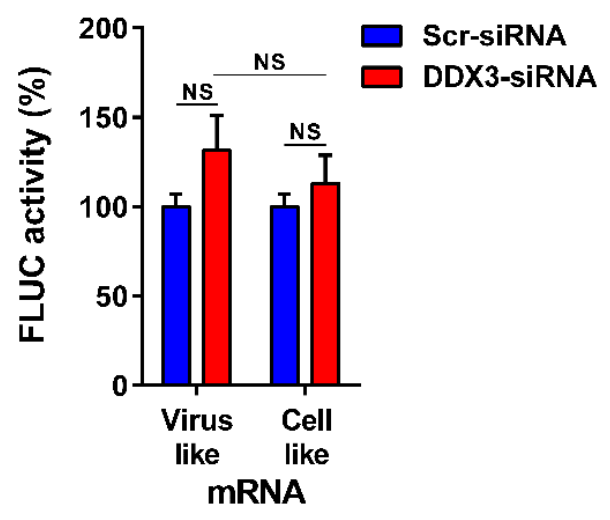

$\mathbf{F}$
B

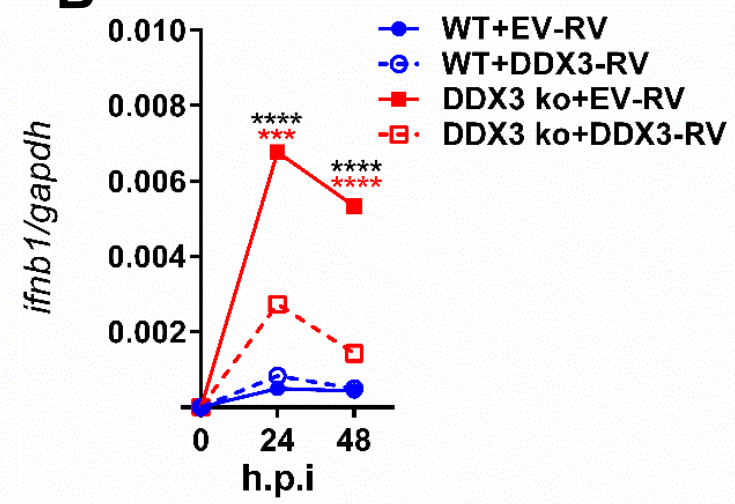

D
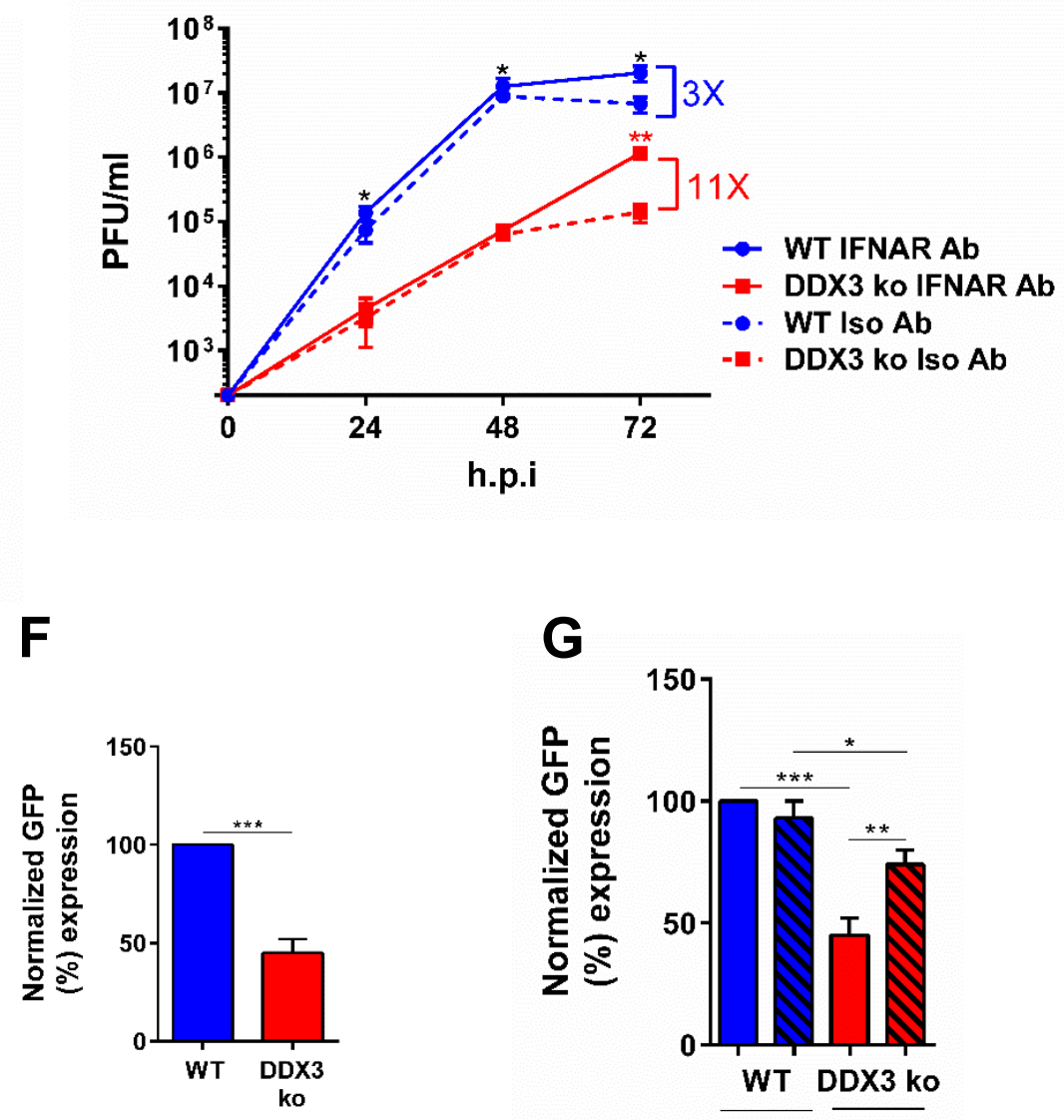

G

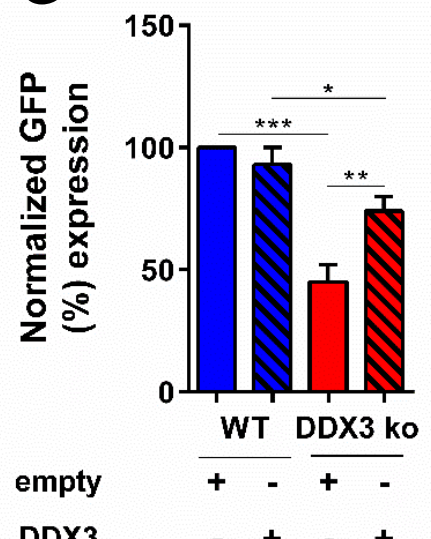


IP: HA

IB: DDX3

IP: -

IB: GAPDH
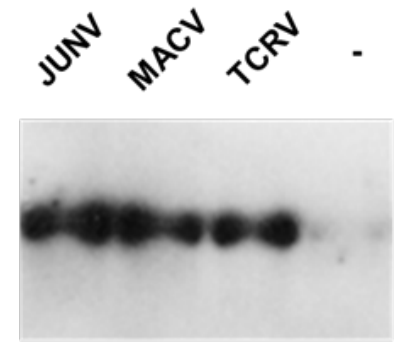

D

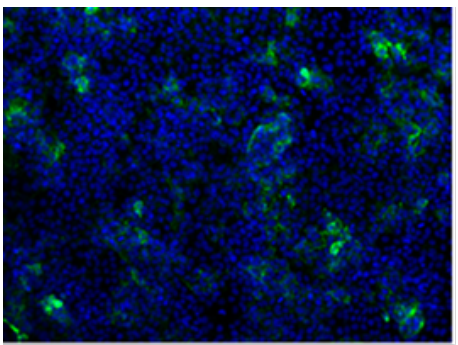

WT

$\mathbf{E}$

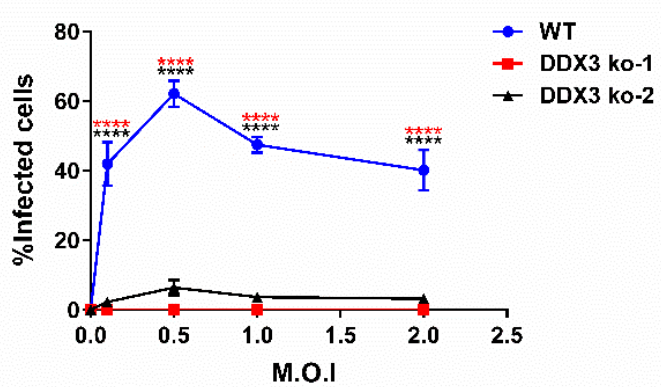

B

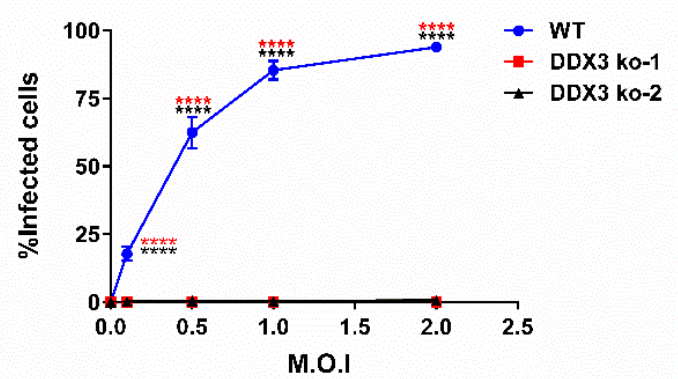

C

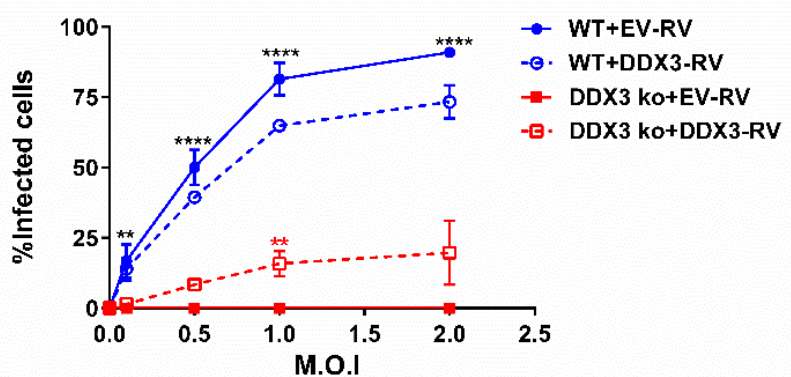

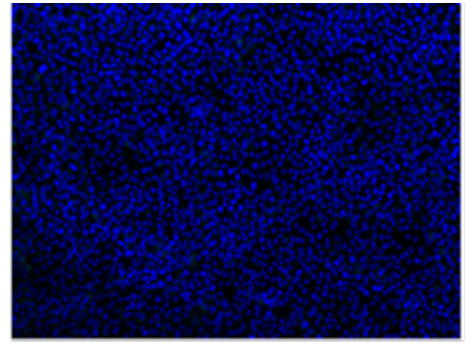

DDX3 ko-1

F

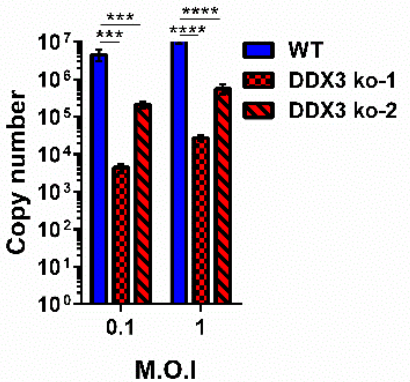

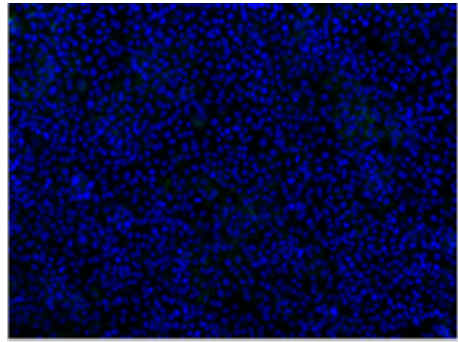

DDX3 ko-2

G

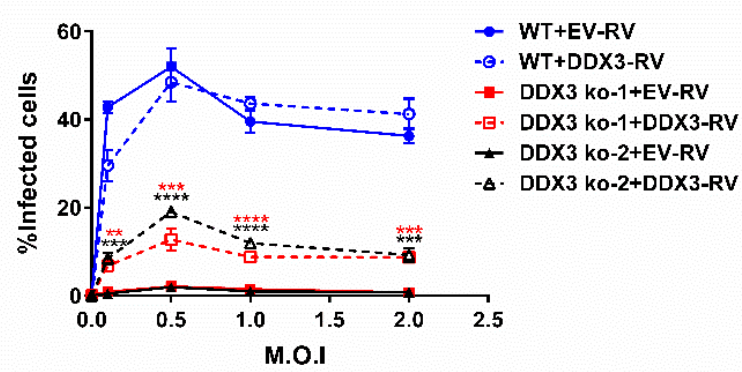

